\title{
BLE-based floor positioning method for multi-level atrium spatial environments
}

\author{
Hongxia $\mathrm{Qi}^{1,2}$ - Yunjia Wang ${ }^{1,2} \cdot$ Jingxue $\mathrm{Bi}^{3} \cdot \mathrm{Hongji}^{\mathrm{CaO}}{ }^{1,2} \cdot$ Shenglei $\mathrm{Xu}^{1,2}$. \\ Hui Liu ${ }^{1,2}$
}

Received: 30 July 2020 / Accepted: 31 May 2021 / Published online: 12 June 2021

(c) Akadémiai Kiadó 2021

\begin{abstract}
Nowadays, cities and towns all over the world are dotted with high-rise buildings, and floor positioning technology has gradually become the focus of indoor positioning research. Multi-level buildings with an atrium space structure mainly consist of shopping malls and hospital outpatient buildings. This kind of building is widely spread, and they usually have a lot of people and a high demand for location-based services. The specific spatial structure of the indoor atrium makes the propagation of wireless signals different, which leads to most of the wireless signal-based floor positioning methods not working well in such an environment. Aiming at this atrium structure environment, this paper proposes a high-precision multi-method adaptive weighted fusion algorithm based on Bluetooth Low Energy signals. In experiments, the real-time dynamic floor positioning accuracy reached $92.7 \%$, while the floor positioning accuracy in the 5-s static state was as high as $100 \%$. Besides, when using $3 \mathrm{~s}$ of test data rolled back, real-time effects could also be achieved, with an accuracy of $98.3 \%$. This method can be used alone for real-time floor positioning, it is suitable for wireless fidelity signals, and it can provide a highly accurate initial floor reference for barometric or inertial methods. It could also be integrated with other methods to provide highly accurate floor locations for long periods of time. The fingerprint library acquisition in this method saves time and effort. The database volume is small and generalizable to a wide range of applications.
\end{abstract}

Keywords Indoor positioning $\cdot$ Floor positioning $\cdot$ BLE signal $\cdot$ Wi-Fi signal $\cdot$ Multi-level atrium space

Yunjia Wang

wyjc411@163.com

1 National Administration of Surveying, Mapping and Geo-Information (NASG) Key Laboratory of Land Environment and Disaster Monitoring, China University of Mining and Technology, Xuzhou 221116, Jiangsu Province, China

2 School of Environment Science and Spatial Informatics, China University of Mining and Technology, Xuzhou 221116, Jiangsu Province, China

3 College of Surveying and Geo-Informatics, Shandong Jianzhu University, Jinan 250101, China 


\section{Introduction}

Applications, such as the Internet of things, location-based services (LBS), and artificial intelligence, cannot be realized without positioning technology. People live indoors most of the time, and indoor positioning technology can fill the gap left by satellite positioning technology in indoor environments like tunnels and buildings. Location-based services make it easier for people to perform a variety of daily operations, including convenient shopping and searching for family or friends at shopping malls, smart parking and car search, airport boarding and pick-up guidance, hospital guidance, and smart storage logistics. With the assistance of indoor positioning technology, services like emergency and rescue, fire rescue, personnel alarm and rescue, crowd monitoring, and emergency guidance will be more accurate and efficient. With the socio-economic development and the increasing number of high-rise buildings, people's demand for indoor positioning has long been needed for multi-level three-dimensional space, that is, multi-level indoor positioning services (Deng et al. 2012; Maneerat et al. 2014a). In most cases, people use floor markers to directly access the floor location. However, in a larger areas or multi-floor environments, floor markers are not visible everywhere. Positioning terminals cannot easily "see" the floor label, especially when there are blind visual limitations or during emergencies, and floor locations are difficult to access. In multi-floor indoor environments, floor identification is even more important in the field of indoor positioning. In multi-floor indoor spaces, to obtain the specific location of the target, the location of the floor must be known before you can further determine the specific location of the target and recall the map of the floor to display the location ( $\mathrm{Li}$ et al. 2014). Floor positioning is particularly useful in emergencies (Gu et al. 2019). At the scene of a fire, timely and accurate access to the floor of the people to be rescued can greatly enhance the overall rescue speed and the chances of saving their lives. On October 30, 2019, the Federal Communications Commission (FCC) issued a draft order. The draft requires that wireless carriers should provide vertical (or z-axis) location data, which for 80 percent of indoor 911 calls must be within $3 \mathrm{~m} \mathrm{(m)} \mathrm{from}$ the user's cell phone ${ }^{1}$. On March 20, 2020, a 28-year-old Chinese Ph.D. student living in the U.S. called 911 for help because his condition was worsening from the coronavirus, but the alarm system showed two possible buildings, and the target building and floor as well as the room information could not be identified, which led to a subsequent unsuccessful search by five police officers, three firefighters, and one search and rescue dog. The caller eventually died in the room ${ }^{2}$. Such cases demonstrate the importance of both indoor positioning and floor positioning. During the outbreak of the COVID-19 in more than 200 countries worldwide, indoor positioning services can be used to search for and identify people suspected of being infected in crowd trajectories. Moreover, the precise location of floors also allows for more accurate search results.

Floor identification can be achieved through a variety of methods or techniques, the main ones being detecting floor changes using barometric pressure changes ( $\mathrm{Li}$ et al. 2014; Xia et al. 2015; Ye et al. 2016; Bai et al. 2013; Retscher 2007), using a wireless signal to decode the target floor (Liu et al. 2017; Razavi et al. 2015; Liu et al. 2012; Campos et al. 2014; Alsehly et al. 2011; Gansemer et al. 2009; Lohan et al. 2015; Maneerat et al. 2014b; Wang et al. 2010), identifying floor changes using motion sensor data (Ye et al.

\footnotetext{
1 https://www.ithome.com/0/453/396.htm.

${ }^{2}$ https://new.qq.com/omn/20200301/20200301A0I3RT00.html.
} 


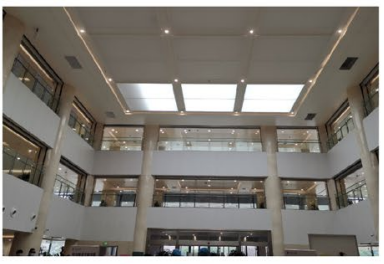

(a) Hospital

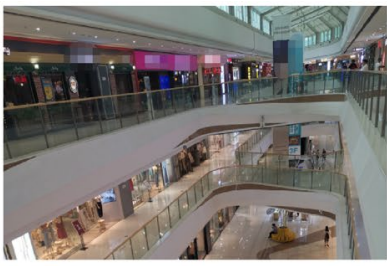

(b) Shopping Mall

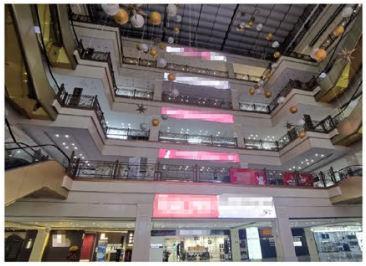

(c) Home Plaza

Fig. 1 Photographs of the inside of multi-floor buildings with an atrium space

2012, 2014; Xu et al. 2017; Fetzer et al. 2018; Ramana et al. 2016), and the realization of floor positioning by multi-method fusion (Ramana et al. 2016; Ye et al. 2014; Gupta et al. 2014; Li et al. 2018; Ashraf et al. 2019; Haque et al. 2018; Elbakly et al. 2018b; Peral-Rosado et al. 2015). Since air pressure is related to elevation and changes in elevation can be inferred from changes in air pressure, changes in floor level can be inferred from the floor height (Li et al. 2014; Xia et al. 2015; Bai et al. 2013). However, the method of positioning floors based on barometric pressure requires an initial floor reference or the use of a barometric pressure reference from a base station (Maneerat et al. 2014a). Different models of barometric pressure sensors take different barometric pressure values at the same location and time. That means different terminals that use the base station to monitor floor changes need to be calibrated in advance ( $\mathrm{Li}$ et al. 2014; Xia et al. 2015). As a result, these methods are expensive to deploy and have limited calibration accuracy and end-use models, making them difficult to apply universally. Similarly, by calculating motion states and relative elevation data, high-frequency data from motion sensors can be used to detect floor changes. Such methods still require the initial floor information to define the absolute floor position (Ye et al. 2012; Fetzer et al. 2018; Ramana et al. 2016). Initial floor determination plays a significant role in the field of floor positioning. Using the propagation characteristics of the wireless signal and the relevant algorithm, the test signal is matched with the corresponding fingerprint library to calculate the final floor result (Deng et al. 2012; Maneerat et al. 2014a; Qi et al. 2019; Abd Rahman et al. 2014). Wireless signalbased floor determination does not require additional set up and it has a high degree of general applicability. Furthermore, it can be used independently or with other methods for floor positioning. For example, it can provide the initial floor information for a barometric pressure-based or motion sensor-based method that can obtain readily available floor level information (Gu et al. 2019; Ramana et al. 2016; Li et al. 2018). All the initial floor positioning methods, especially those based on wireless signals, are indispensable in the field of floor identification and indoor positioning.

Atrium structures are quite common in multi-level shopping malls or plazas as well as in the outpatient buildings of major hospitals as they allow people to obtain information about the functions or departments on each floor at a glance as well as provide a wider view along with good experience and convenient service. According to Baidu Encyclopedia, the atrium space is a form of architectural space, which refers to the courtyard space inside a building and is characterized by the formation of an "outdoor space" that is located 
Table 1 Overview of multi floor indoor structures based on a wireless signal for floor positioning

Table 1 Overview of multi-floor indoor structures based on a wireless signal for floor positioning

\begin{tabular}{|c|c|c|c|}
\hline Literature & Indoor floor plan & Literature & Indoor floor plan \\
\hline $\begin{array}{l}\text { (Maneerat et al. } \\
\text { 2014a) }\end{array}$ & (E) & (Qi et al. 2019) & \\
\hline (Liu et al. 2017) & & $\begin{array}{c}\text { (Abd Rahman et al. } \\
\text { 2014) }\end{array}$ & 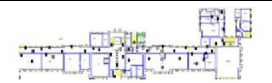 \\
\hline (Razavi et al. 2015) & & (Alshami et al. 2017) & $-{ }_{-1}^{-}-$ \\
\hline (Razavi et al. 2015) & & (Bhargava et al. 2012) & $y=6$ \\
\hline (Liu et al. 2012) & & (Zhang et al. 2018) & 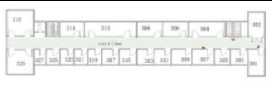 \\
\hline (Campos et al. 2014) & & (Razavi et al. 2016) & \\
\hline (Alsehly et al. 2011) & $\sqrt{=}$ & (Sun et al. 2015) & 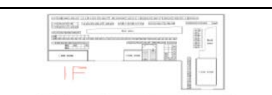 \\
\hline (Lohan et al. 2015) & & (Sun et al. 2015) & $\sqrt{1-i}$ \\
\hline $\begin{array}{c}\text { (Maneerat et al. } \\
2014 b)\end{array}$ & & (Han et al. 2019) & 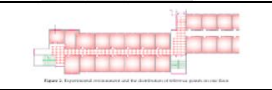 \\
\hline (Wang et al. 2010) & $22 \div-\frac{1}{25}=$ & (Maneerat et al. 2016) & \\
\hline (Ramana et al. 2016) & 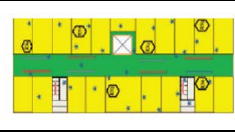 & $\begin{array}{c}\text { (Elbakly et al. 2018a; } \\
\text { Zheng et al. 2016; } \\
\text { Zhao et al. 2017) }\end{array}$ & $\begin{array}{l}\text { No indoor plane or } \\
\text { test site pattern }\end{array}$ \\
\hline (Ye et al. 2014) & 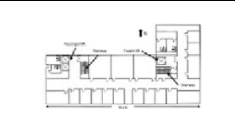 & $\begin{array}{c}\text { (Elbakly et al. 2020) } \\
\text { and (Razavi et al. } \\
\text { 2016) }\end{array}$ & \\
\hline
\end{tabular}

inside the building ${ }^{3}$. It is a kind of shared space formed by the upper and lower floors of the building, and some indoor scenes are shown in Fig. 1. Such buildings are now very common. Wireless signals have different propagation characteristics in different multi-floor indoor layout environments (Honcharenko et al. 1993; Alshami et al. 2017; Shi et al. 2013). In the atrium area, the signal attenuation is greatly reduced by the lack of a floor plate to block the signal. The attenuation of the signal across floors is not evident in this environment. Consequently, the effectiveness of most wireless signal-based floor location methods in such environments is degraded by changes in signal characteristics Shi and Shin (2013).

Multi-floor buildings with atrium space are widely distributed, mainly in shopping malls and hospitals all over small, medium, and large cities. In such environments, where people are densely distributed, there is usually a strong demand for LBS. Thus, achieving

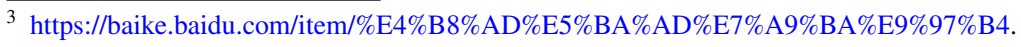


high-precision floor positioning in these environments is of great research value. Currently, there is very little research devoted to floor positioning and especially initial floor positioning in terms of such environments. In our paper, the vast majority of the literature on floor positioning based on wireless signals is summarized and organized. Moreover, the interior plans of the floor structures of the test site in the literature are summarized and shown in Table 1. As shown, there are very few floor positioning experiments in the literature based on wireless signals that have been carried out specifically for atrium spaces. The second test site in the literature (Razavi et al. 2015) had an atrium space, but since the experimental area was separated from the atrium space by offices and floor plates, there was not an experiment in a multi-floor building with an atrium space.

In the study of algorithms related to wireless signal-based floor positioning, each method performs well in the experimental environment in which it is located. However, the peculiarities of the multi-floor environment of the atrium space result in most methods being less accurate. A Wireless Local Area Network (WLAN) floor determination method based on K-means clustering was introduced by Deng et al. (2012). Their methodology is based on the idea that the same wireless fidelity (Wi-Fi) access point (AP) has significant variation in Received Signal Strength Index (RSSI) from floor to floor. Such methods are effective in general multi-floor environments, especially in large-scale multi-floor indoor spaces, such as airports. However, in a multi-level space with an atrium structure, the changes of RSSI of the same AP on different floors are not obvious, and the signal similarity is very high. Therefore, a method based on K-means does not work well for the floor determination for the type of environment studied in this paper. Maneerat et al. (2014a) focused on the usage of the sum-RSSI floor algorithm for floor determination. This method eliminates the need to prepare for the fingerprint library in advance and determines the floor using RSSI from the real-time collection. The RSSI outliers are removed first, and then the RSSI averages of all APs are aggregated. After that, the floor on which the AP group corresponding to the maximum summary average RSSI value is located is finalized as the target floor. This method is simple to implement. However, in an atrium structure space, in addition to meeting the condition of having the same number of APs, a better effect can be achieved when the APs are evenly distributed on each floor. Moreover, the AP signal of other floors is often stronger than the AP signal of this floor, and this often leads to the vulnerability of floor positioning to errors. The nearest neighbor floor algorithm was used by Alsehly et al. (2011). The application of this method requires the creation of a more complete training sample in the early stages. The Wi-Fi signal similarity between multiple floors based on the atrium space is relatively high, and the wireless signal is easily affected by the layout of the indoor atrium, human activity, and so on. This results in AP signals on other floors being stronger than those on the current floor, which ultimately leads to erroneous results. The training samples also require a great deal of labor and resources to collect and continuously maintain to ensure excellent floor positioning accuracy. The other group variance algorithm, while having better site coverage, is less accurate than the nearest neighbor floor algorithm. Therefore, in multi-floor environments with an atrium space, the high signal similarity results in a poor floor positioning effect. The floor signal interval confidencebased algorithm (Qi et al. 2019) is more suitable for indoor environments with large areas and multiple floors, where the AP location is unknown and unevenly distributed. This method provides a better positioning result and the fingerprint collection process not only saves time but also effort. However, in the multi-floor environment of the atrium space, the signal difference between floors is minimal due to the long distance of Wi-Fi signal propagation, which makes it difficult to generate an effective signal interval to distinguish the signal characteristics of different floors. If the signal interval is too large, then it is not 
sufficient to distinguish floors; while if it is too small, it is difficult to adapt to changes in the indoor environment, such as human activity. The method, therefore, works worse in multi-floor environments with an atrium space than in regular multi-floor environments. The idea of floor positioning by Zhang et al. (2018) is based on the apparent difference in Wi-Fi signal strength between floors. However, in the indoor structure of the atrium space, the difference in Wi-Fi signal between adjacent floors is not obvious, and thus it is easy to tend to misidentify the floor information.

A Bayesian classification with a Wi-Fi signal-based floor identification algorithm (BCFI) was proposed by Zhao et al. (2017). During floor identification using a Wi-Fi signal, when a Wi-Fi signal propagates through a floor made of mat reinforcement concrete, the signal results in a sudden and remarkable attenuation (e.g., $50 \mathrm{~cm}$ thick concrete causes about $29 \mathrm{dBm}$ attenuation). However, this feature does not exist in a multi-floor environment with an atrium space. The BCFI algorithm uses the floor with the largest posterior probability as the final floor estimation. The BCFI algorithm has good performance in terms of accuracy and robustness compared with SkyLoc (Alex et al. 2007) and clustering algorithms. This paper showed that in the floors with large hollow areas, the BCFI algorithm has a relatively small RSSI difference between adjacent floors, which leads to a significant reduction in floor recognition accuracy. At this time, the average accuracy of floor recognition of the test data only exceeds $15 \%$, which is insufficient. Furthermore, another study (Zhao et al. 2017) introduced the BPFI algorithm for floor identification based on barometric pressure. The premise of this algorithm is that, after manual input or high-confident floor estimation is used for initialization, the pressure difference can be used to determine the relative floor transition to finally complete the floor identification. If there is no high-precision initial floor position, then the pressure-based floor positioning results will be unreliable. Depending on the ubiquitous Wi-Fi signals and AP locations, the StoryTeller method (Elbakly et al. 2020) can be employed to generate images, build the virtual architecture, train the model by using a convolutional neural network (CNN), and finally obtain a higher precision result. The basic idea of this method is that APs on the target floor experience less attenuation and stronger RSSs are generally received from APs on the target floor. This method depends on the relative location of the AP, and it is difficult to obtain the location in reality. Meanwhile, this method performs well in ordinary buildings with concrete floor slabs. However, for the multi-story environment of the atrium spaces, the wireless signal propagation characteristics are different (Chapter 2.6), which affects the performance of this method. Moreover, Huang et al. (2020) proposed a floor positioning method for hollow areas. This method combines a variety of sensors to achieve high-precision floor positioning. At the same time, the study also points out that the Wi-Fi fingerprints between adjacent floors are very similar and it is difficult to accurately localize floors. Therefore, it is difficult to accurately identify the floors using only the Wi-Fi-based floor positioning method. In hollow areas, the barometric pressure-based floor positioning method with "reference barometric pressure" is used to predict the floor. This method requires additional deployment and data communication development, which is costly and not conducive to popularization and application.

In conclusion, in the multi-floor structure of the atrium space, the signal differences between floors are not obvious. Consideration can be given to combining the RSSI distribution of the wireless signal and the deployment of APs to carry out floor positioning from a multi-angle analysis of the signal characteristics, the number of APs, RSSI mean value, and so on, which can effectively improve the accuracy of floor positioning in such environments and realize high-precision results. In summary, most of the initial floor or floor positioning methods based on wireless signals have their advantages in common 
multi-floor environments, but few have been tested and verified specifically in multifloor indoor environments with atrium space structures. Based on the above analysis and the analysis of the test of wireless signals in a multi-floor environment with an atrium space in Chapter 2, it was found that the signal of the Bluetooth Low Energy (BLE) has a shorter transmission distance than a Wi-Fi signal. In the test environment, the signal and AP coverage of BLE on different floors was significantly lower than that of Wi-Fi, indicating that BLE has more obvious floor differences, and thus a BLE signal is more suitable for high-precision floor positioning in such environments. Bluetooth Low Energy modules are commonly used today in the intelligent world, and their main applications include location tags, asset tracking, sports and health sensors, medical sensors, smartwatches, remote controls, toys, and so on, with a certain universality. Moreover, in the Internet of Things era, the BLE module has become one of the indispensable supporting modules. In practical applications, BLE modules are inexpensive, easy to deploy, and have a certain universality. A multi-floor environment with an atrium space is not an exception, and it is widely distributed with a high volume of indoor traffic, making floor positioning in such an environment extremely useful. This paper proposes a hybrid method for floor positioning using smartphones and BLE signals specifically for indoor multi-floor environments in atrium spaces. Two methods are used to calculate the results of the respective floors, assign adaptive weights to the different results, and then combine the two results with the normalized weights to make a combined decision and then resolve the information of the target floor. The method performs well in the multi-floor environment of the atrium space, with a low fingerprint database collection effort and low cost, making it easy to apply for general use by the public.

The subsequent chapters are as follows: Chapter 2 describes the spatial distribution characteristics of wireless signals in a multi-floor environment with indoor atrium spaces and compares the differences between Wi-Fi and BLE signals. Chapter 3 introduces the proposed algorithm. Chapter 4 carries out relevant experiments and validations. Finally, Chapter 5 provides the final summary and perspectives of the main theme.

\section{Spatial distribution characteristics of wireless signals in a multi-floor indoor atrium environment}

\subsection{Introduction to the test site}

The test site was located at the National Key Laboratory of Navigation Satellite System and Equipment Technology, the 54th Research Institute of China Electronics Technology Group Corporation (CETC54), Shijiazhuang, Hebei Province, China. In this paper, it is referred to as the $\mathrm{C} 7$ test site. The $\mathrm{C} 7$ test site has three floors of unequal heights, with a typical atrium space structure inside. Each floor is about $28 \mathrm{~m}$ long and $25 \mathrm{~m}$ wide, covering an area of about 700 square $\mathrm{m}$. The test site is equipped with a variety of equipment and devices specifically designed for indoor positioning research, including BLE, Wi-Fi, cameras, pseudo-satellite, and so on. The specific test environment is shown in Fig. 2. The blue and red triangles in the figure show the location of the BLE installation on the three floors, where the brand of the BLE is Yunli 'uli E5, priced at 45 Chinese Yuan per unit. Floors 1-3 of the test site were equipped with 18, 18, and 21 BLE devices and 26, 30, and $20 \mathrm{Wi}-\mathrm{Fi}$ devices. 
Fig. 2 The C7 test site environment and the BLE layouts (red and blue triangles indicate the locations of the BLE devices)

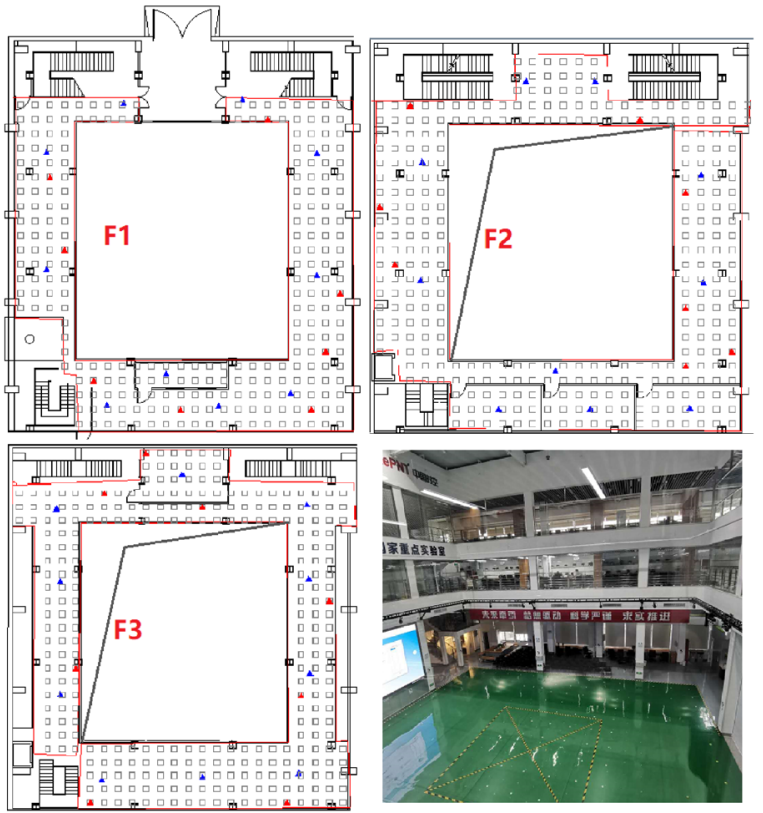

Table 2 Example format of Wi-Fi/BLE FFPDB

\begin{tabular}{lllllll}
\hline Floor_id & Test_id & MAC_id & $\operatorname{RSSI}_{1}(\mathrm{dBm})$ & $\operatorname{RSSI}_{2}(\mathrm{dBm})$ & $\ldots$ & RSSI $_{\mathrm{n}}\left(\mathrm{dBm}^{2}\right)$ \\
\hline F1 & 01 & C2:02:8E:00:00:71 & -93 & -85 & $\ldots$ & -85 \\
F1 & 01 & C2:02:8E:00:00:73 & -81 & -82 & $\ldots$ & -82 \\
$\ldots$ & $\ldots$ & $\ldots$ & $\ldots$ & $\ldots$ & $\ldots$ & $\ldots$ \\
F1 & 02 & C2:02:8E:00:03:1A & -70 & -77 & $\ldots$ & -76 \\
$\ldots$ & $\ldots$ & $\ldots$ & $\ldots$ & $\ldots$ & $\ldots$ & $\ldots$ \\
F3 & 19 & C2:02:8E:00:03:1B & -78 & -82 & $\ldots$ & -83 \\
$\ldots \ldots$ & & & & & &
\end{tabular}

\subsection{Introduction of Wi-Fi/BLE signal acquisition software and experimental data}

We used the same signal acquisition software like the one in prior work (Bi et al. 2018), which was installed on a smartphone, and with an acquisition frequency of $1 \mathrm{~Hz}$. The software could collect and save the RSSI value of every second of all the APs of BLE and Wi-Fi that could be searched within a set period of time. We set up two types of methods for signal acquisition. One was static acquisition where the position and posture of the mobile handsets were kept constant during acquisition, also known as standing acquisition. Another type of acquisition was the dynamic acquisition in which the position or attitude of the phone constantly changed during the acquisition process, also known as walking acquisition. The fingerprint library of the floors was chosen in the same way as (Qi et al. 2019), namely, slow walking to collect the Wi-Fi/BLE signals of each floor, while generating the interval confidence floor fingerprint library (ICFDB). Besides, there was a test point every 3-4 $\mathrm{m}$ on each floor, and the static signal was collected to generate a floor fixed-point 
Table 3 Example format of Wi-Fi/BLE FDTDB

\begin{tabular}{llll}
\hline Floor_id & Test_tid & MAC_List & RSSI $_{1}(\mathrm{dBm})$ \\
\hline F1 & 001 & C2:02:8E:00:00:71 & -93 \\
F1 & 001 & C2:02:8E:00:00:73 & -81 \\
$\ldots$ & $\ldots$ & $\ldots$ & $\ldots$ \\
F2 & 001 & C2:02:8E:00:03:1A & -70 \\
$\ldots$ & $\ldots$ & $\ldots$ & $\ldots$ \\
F3 & 109 & C2:02:8E:00:03:1B & -78 \\
F3 & 109 & C2:02:8E:00:03:2C & -79 \\
$\ldots \ldots$ & & & \\
\hline
\end{tabular}

Table 4 Example format of $\mathrm{Wi}-\mathrm{Fi} / \mathrm{BLE}$ APFDB

\begin{tabular}{ll}
\hline Floor_id & MAC_id \\
\hline F1 & C2:02:8E:00:00:71 \\
F1 & C2:02:8E:00:00:73 \\
$\ldots$ & $\ldots$ \\
F2 & C2:02:8E:00:03:1A \\
$\ldots$ & $\ldots$ \\
F3 & C2:02:8E:00:03:1B \\
$\ldots \ldots$ & \\
\hline
\end{tabular}

database of fingerprints (FFPDB). Then, a walk was done around each floor and signal data per second was collected to generate a library of floor dynamics tests (FDTDB). After that, the layout information of each AP is collated and the AP floor location library was generated (APFDB). The format of the ICFDB from prior work (Qi et al. 2019) and other database formats are shown in Tables 2, 3, 4 .

\subsection{Spatial distribution characteristics of Wi-Fi/BLE signals in multi-floor atrium spaces}

In indoor multi-floor environments with an atrium space, the wireless signal becomes more "unobstructed" to travel through space without blocking from either floor plates or the corresponding walls in the atrium area, and there is a higher signal similarity between adjacent floors. To analyze the Wi-Fi/BLE FFPDB, FDTDB, and APFDB, and to display the distribution of the signal at each TP of the APs on each floor from the 1 st to the 3rd floor, the 5-s Wi-Fi/BLE static signal distribution is shown in Fig. 3, while the 1-s BLE real-time dynamic signal distribution is shown in Fig. 4. There are 67 TPs in Fig. 3, of which 30 are on the first floor, 19 on the second floor, and 18 on the third floor; and there are 275 TPs in Fig. 4, of which 90 are on the first floor, 95 on the second floor, and 90 on the third floor. Figures 3 and 4 are presented in a two-dimensional table, where the yellow, green, and orange areas represent different Wi-Fi/BLE APs or TPs on floors 1-3, respectively. The data section shows the strength of the signal in a red gradient; the darker the color, the stronger the signal. Different tables from left to right represent the distribution of signals 

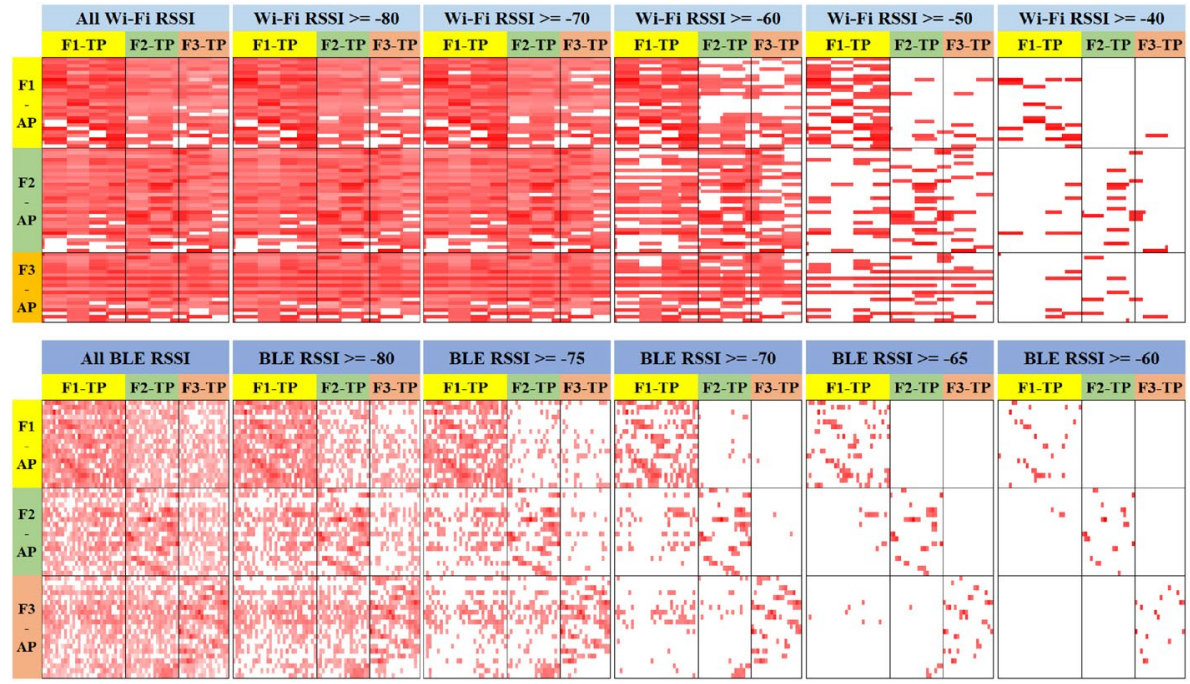

Fig. 3 The RSSI distribution of Wi-Fi/BLE for 5-s static signals at all the TPs on each floor, respectively

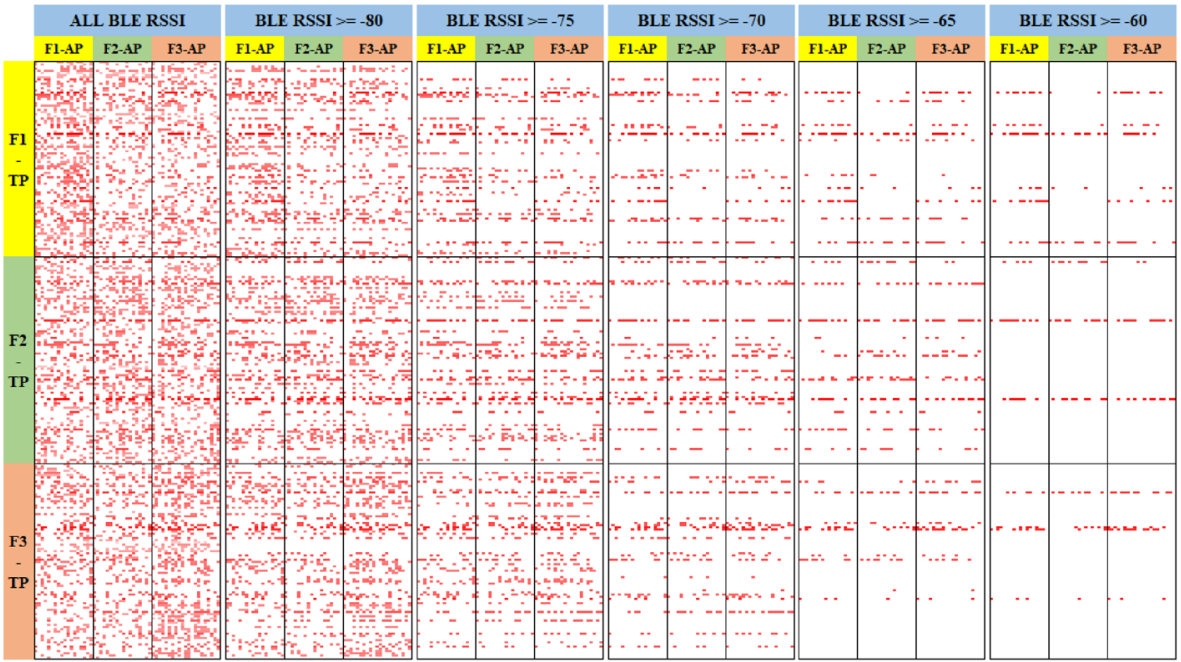

Fig. 4 RSSI distribution of BLE for the 1-s dynamic real-time signal at all TPs on each floor

in different RSSI ranges, for example, all the signal values, $\operatorname{RSSI} \geq-80, \operatorname{RSSI} \geq-70$, RSSI $\geq-60$, and so on.

Figure 3 shows that Wi-Fi had stronger signal coverage and RSSI than BLE. In the multi-floor environment of the atrium space, the Wi-Fi signal had high similarity in the adjacent floors, making it difficult to effectively identify the floors with the Wi-Fi signal distribution characteristics. The BLE signal propagation distance was short, and the RSSI of the BLE AP which was at the deployment floor was significantly better than that of other floors when the BLE signal was greater or equal to $-70 \mathrm{dBm}$. The performance was more 
Fig. 5 The Wi-Fi/BLE AP signal coverage rate at the current floor, adjacent floors, and two floors apart

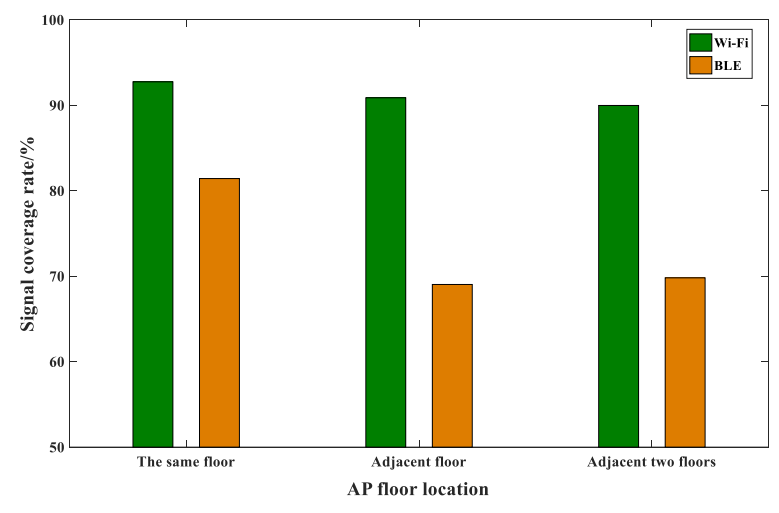

prominent when the signal was greater or equal to $-60 \mathrm{dBm}$, which is when the signal distribution has stronger floor properties. As a result, the short transmission distance and strong floor signal properties of BLE signals are highlighted. Both these properties indicate that BLE signals are more suitable than Wi-Fi for conducting floor positioning in such environments. The comparison of the BLE signal data in Figs. 3 and 4 shows that the static fixed-point data has better floor properties and high signal coverage as well as good signal stability. The 1-s real-time dynamic data has weak floor attribution characteristics as well as low signal coverage and poor signal stability. Therefore, from the distribution characteristics of each signal, only using RSSI as an eigenvalue to calculate the floor location accuracy is not reliable enough. Therefore, it is necessary to consider both RSSI and AP layout information as the eigenvalue to improve the floor positioning accuracy.

\subsection{Wireless fidelity and BLE signal coverage at different floors of the atrium space}

In the test site, the Wi-Fi/BLE FFPDB contained $76 \mathrm{Wi}-\mathrm{Fi}$ and $57 \mathrm{BLE}$ APs and as well as 67 TPs. Figure 5 shows the Wi-Fi/BLE AP signal coverage rate at the current floors, adjacent floors, and two floors apart. There is an example of Wi-Fi signal coverage rate on the same floor (which is the corresponding data of the left dark green bar chart in Fig. 5). The numerator is cumulatively derived from the number of signal frequencies at the TPs of all the floor APs of the respective floors, and the denominator is derived from the total number of frequencies in the full distribution of the number of APs in all floors in the TPs of the respective floors, and the ratio of the two is the signal coverage of the same floor. Figure 5 shows that, in the test site environment, the coverage of the BLE signal on the current floor was only about $80 \%$, with less than $70 \%$ coverage on all other floors. Thus, the BLE signal coverage was significantly weaker than the Wi-Fi signals. This illustrates that the BLE signal was less similar in different floors, and the index of differentiating floors performed better than the Wi-Fi signal.

When the above test data were integrated again, the ratio of the number of APs collected from each test site to the total number of test APs was counted, as shown in Fig. 6. The red line in the figure indicates Wi-Fi data, while the blue line indicates BLE data. Each point represents the ratio of the number of APs with the signal at each TP to the total number of test APs, that is, it is the signal coverage of the Wi-Fi and BLE APs at each TP. Most of the Wi-Fi has signal coverage was more than $90 \%$ throughout all of the three floors' TPs. This shows that the Wi-Fi signal had a high similarity across different floors and regions 


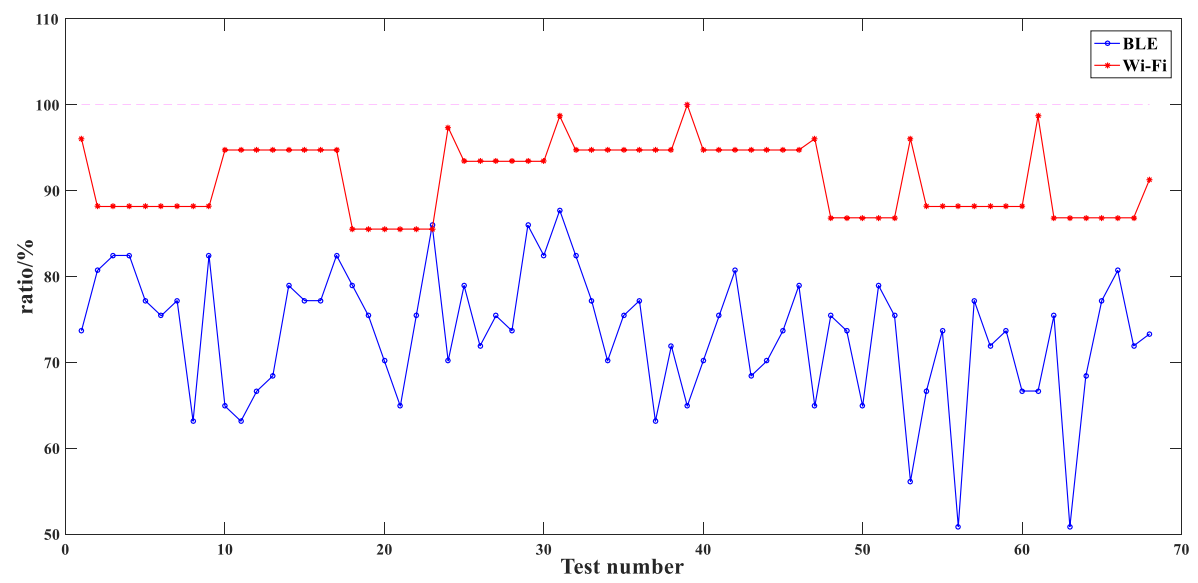

Fig. 6 The AP coverage of Wi-Fi/BLE at all TPs

throughout the test environment, and the floor properties of the signal were not obvious. The BLE data varied, with the number of BLE APs collected at most test sites accounting for only about $70 \%$ of the total. That is, there was about $30 \%$ variability in the AP sequences of BLE for different floors. Therefore, the floor difference of the BLE signal was better than that of Wi-Fi.

The above analysis of Wi-Fi and BLE signals on multiple floors of the atrium space shows that the BLE's floor recognition is stronger than the Wi-Fi signal because the BLE's transmission distance is less than the Wi-Fi signal. Most floor location algorithms based on Wi-Fi signals, however, suffer some degradation in performance in the atrium space environment, making it difficult to meet basic requirements. Therefore, in this research, the BLE signal is used to carry out the floor positioning test and verify the positioning performance according to the peculiarities of a multi-floor environment with an atrium space.

\subsection{Comparison of spatial distribution characteristics of wireless signals in a multi-floor environment with or without an atrium space structure}

A 30-s Wi-Fi and BLE signal was collected at the same horizontal location on all three floors to find the RSSI distribution of the APs that were installed on the third floor. The details are shown in Fig. 7. Figure 7a shows the signal distribution from floors 1-3 of the Wi-Fi APs installed on the 3rd floor in our test site. Figure $7 \mathrm{~b}$ shows the signal distribution from floors 2 to 4 of Wi-Fi APs installed on the 4th floor in a common multi-floor environment (Qi et al. 2019). Figure 7c shows the signal distribution from floors 1-3 of the BLE APs installed on the 3rd floor in our test site. As shown, the Wi-Fi signal attenuation on the adjacent floors was about $25 \mathrm{dBm}$ in the normal full-floor laminate environment, while the attenuation was only about $2 \mathrm{dBm}$ in the atrium space environment. The former value is far greater than the latter. In the multi-floor environment of the atrium space, the Wi-Fi signal did not vary significantly from one floor to the next. This feature greatly affects the accuracy of most floor positioning methods based on Wi-Fi signals. The BLE signal had a shorter propagation distance, and the signal attenuation in the atrium space adjacent to the floor was about $15 \mathrm{dBm}$, which was significantly greater than the value of the Wi-Fi signal. 


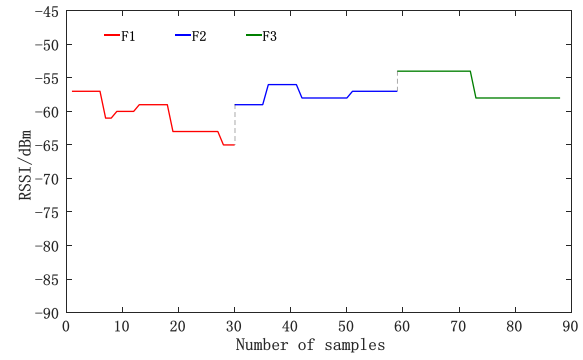

(a) Multi-floor Wi-Fi signal in the atrium space

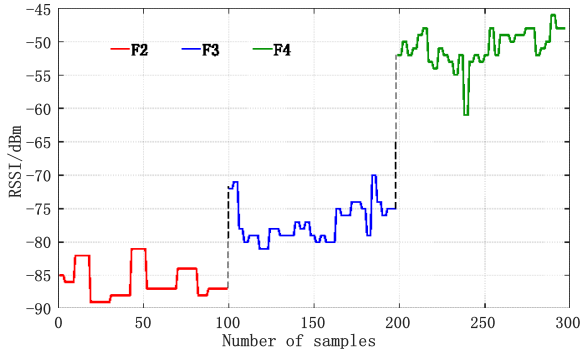

(b) Multi-floor Wi-Fi signal without an atrium space

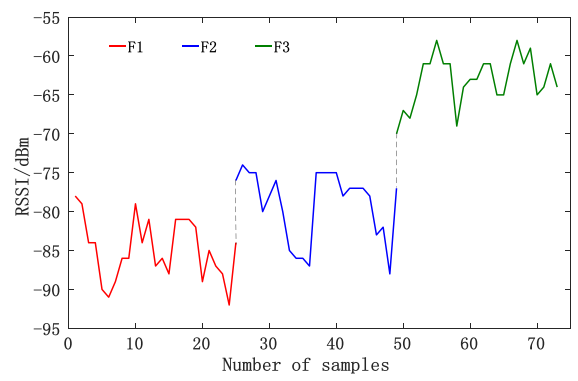

(c) Multi-floor BLE signal in the atrium space

Fig. 7 The RSSI profile of Wi-Fi/BLE signals in the three adjacent floors in multi-floor environments with different indoor structures

Although BLE has poor signal stability, it is still superior to Wi-Fi for floor positioning in the test site environment of this study.

\subsection{Comparative analysis between the floor where the largest RSSI of the AP is located and the actual floor of the AP}

Aiming at the part of the floor identification method based on the location of the AP, the corresponding floor signal was analyzed at the C7 test site, and the floor with the largest RSSI in all APs and the real one installing the APs were extracted and then comparatively analyzed. The results are shown in Fig. 8. In this figure, the calculated floor represents the floor where the AP's strongest signal was located, and the real floor represents the actual floor location of the APs. In the multi-story environment of the atrium space structure, the location of the floor where the AP's strongest signal was located was not necessarily the location of the AP's deployment floor. The Wi-Fi signal propagation distance was relatively long, resulting in only $61 \%$ of the two floors having the same location. Moreover, BLE performed much better, and it accounted for $86 \%$. The theory of laying the floor AP with the strongest signal was weaker in this kind of environment, which easily leads to the failure of the method relying on Elbakly et al. (2020) in the atrium structure environment. The main reason for this phenomenon is that, first, the lack of floor plate greatly reduces the attenuation effect of RSSI, and, secondly, the multipath effect makes the RSSI fluctuate greatly (about $10 \mathrm{dBm}$ ). The transmission distance corresponding to this signal difference $(10 \mathrm{dBm})$ is likely to be greater than the height of the floor. Therefore, it is understandable for this phenomenon to occur in an atrium space environment. 
(a) Wi-Fi-The accuracy is $61 \%$

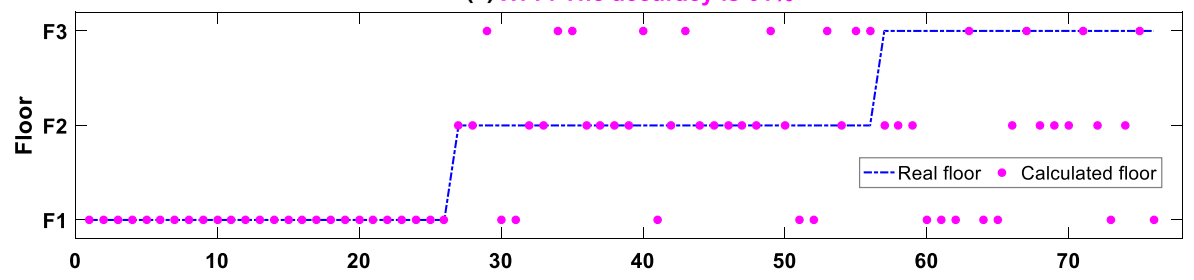

(b) BLE-The accuracy is $86 \%$

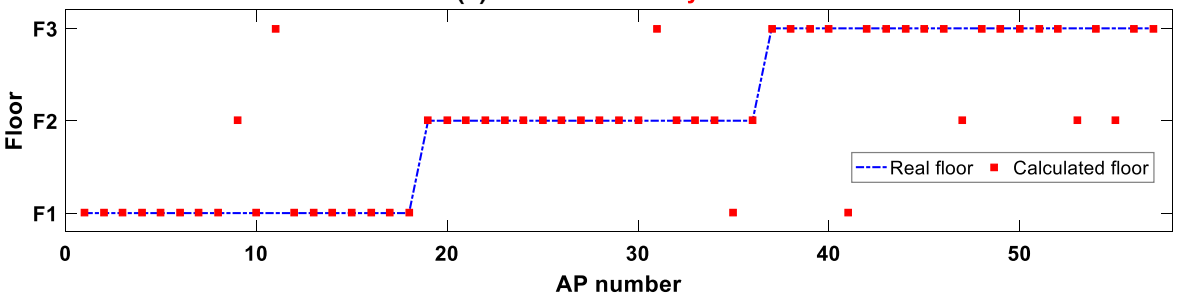

Fig. 8 Atrium space AP real floor and its largest RSSI floor distribution

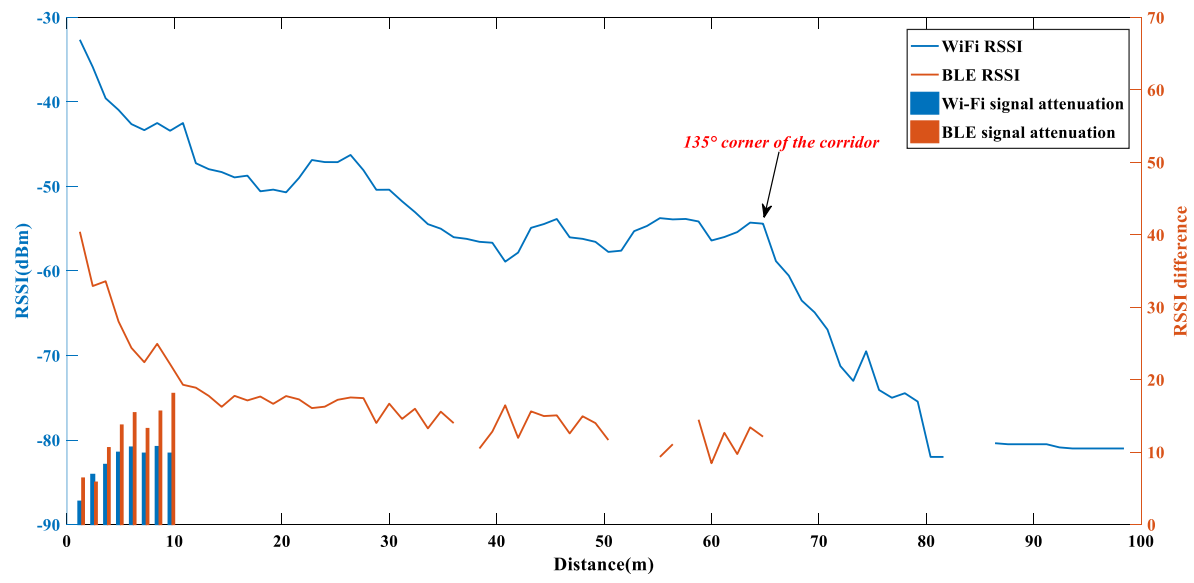

Fig. 9 The RSSI and transmission distance of Wi-Fi/BLE and RSSI attenuation within $10 \mathrm{~m}$

\subsection{The RSSI and transmission distance of Wi-Fi/BLE}

Figure 9 shows the RSSI of the Wi-Fi/BLE signal within $100 \mathrm{~m}$ as the distance increases and the Wi-Fi/BLE RSSI attenuation at a distance of $2-10 \mathrm{~m}$ in the indoor horizontal ground scene. The transmission distance of Wi-Fi signals was greater than that of BLE signals, and the RSSI was stronger than BLE. The signal attenuation of BLE was more significant between $2 \sim 10 \mathrm{~m}$. In the multi-story environment of the atrium space, the signal difference of BLE on different floors was more obvious, and the effect of floor differentiation was better. Through the analysis of the Wi-Fi/BLE signal, the following two propagation characteristics of the wireless signal are reflected: (1) During the propagation process, the RSSI attenuates with the increase of the transmission distance; (2) The multipath phenomenon of the wireless signal causes the fluctuations of the RSSI to be relatively high. 
Furthermore, the propagation distance of BLE is shorter than Wi-Fi, and the signal attenuation of BLE at the same propagation distance is more obvious than Wi-Fi (as can be seen from the signal attenuation within $10 \mathrm{~m}$ shown in Fig. 9). In short, the short transmission distance of BLE signals makes the signal difference between different floors more obvious than Wi-Fi signals, and BLE is better than Wi-Fi when locating floors in this type of environment.

\section{The BLE initial floor positioning method based on multi-algorithm fusion}

The spatial distribution characteristics of BLE signals in multi-floor environments with an atrium space show that the RSSI alone is not sufficient to find the floor position with high accuracy. In this study, the RSSI and AP layout data were incorporated into eigenvalues together and then solved, and a BLE signal-based, multi-method integrated floor positioning algorithm is proposed. The algorithm is divided into an offline stage and an online stage. In the offline stage of this study, we trained three datasets. The first dataset used the same approach proposed by Qi et al. (2019) to capture fingerprints and then generated a database of signal interval confidence fingerprints. The second collected AP set-up information to generate a floor location library. The third collected test data, using two algorithms to generate process data then used them for training to derive two weight normalization factors. The two factors were used for the two methods during the online phase. In the online stage, the BLE test signal is first denoised to generate denoising data. Secondly, the signal interval confidence algorithm (Qi et al. 2019) is used to calculate the sum of confidence levels corresponding to each floor from the denoising data, and then the results of the sum of confidence levels for each floor are saved. Then the floor 1 with the max result is calculated and saved. At the same time, using denoising data and referring to the AP floor position database, the signal averages from the denoising data for each floor are calculated and saved. After that, the floor where the maximum signal average value is located is judged as the result of floor 2, and the signal average value of each floor is saved. Then, based on the two floor results, a weight adaptive normalization process filter is performed. Finally, the estimated value of the floor after the filter is calculated, and the estimated value is rounded to the resulting floor position, which is the final result of the floor information. The specific floor positioning process is shown in Fig. 10.

\subsection{Signal denoising}

The test data was the average signals in several seconds collected at an arbitrary stationary position. It is denoted as $\mathrm{TF}_{\text {test }}=\left\{\mathrm{Mac}_{\mathrm{i}}, \mathrm{rssi}_{\mathrm{i}}\right\}(\mathrm{i}=1,2,3, \ldots, \mathrm{n})$, where $\mathrm{Mac}_{\mathrm{i}}$ is the media access control (MAC) address of the $\mathrm{i}$-th BLE and $\mathrm{rssi}_{\mathrm{i}}$ is the corresponding average rssi for the ith BLE. How much data needs to be removed for weak signals should be based on the training and positioning tests in the test site environments. Our multiple tests suggested that BLE signals weaker than $-90 \mathrm{dBm}$ induce noticeable interference with the following floor positioning algorithms. Because the floor difference of this part of the signal is weak, the corresponding AP cross-floor property is obvious, and this characteristic can be seen in Figs. 3 and 4. Therefore, the real-time test signals with RSSI smaller than $-90 \mathrm{dBm}$ were considered to be noise and were removed to improve the floor positioning accuracy. The signal denoising equation is as follows: 


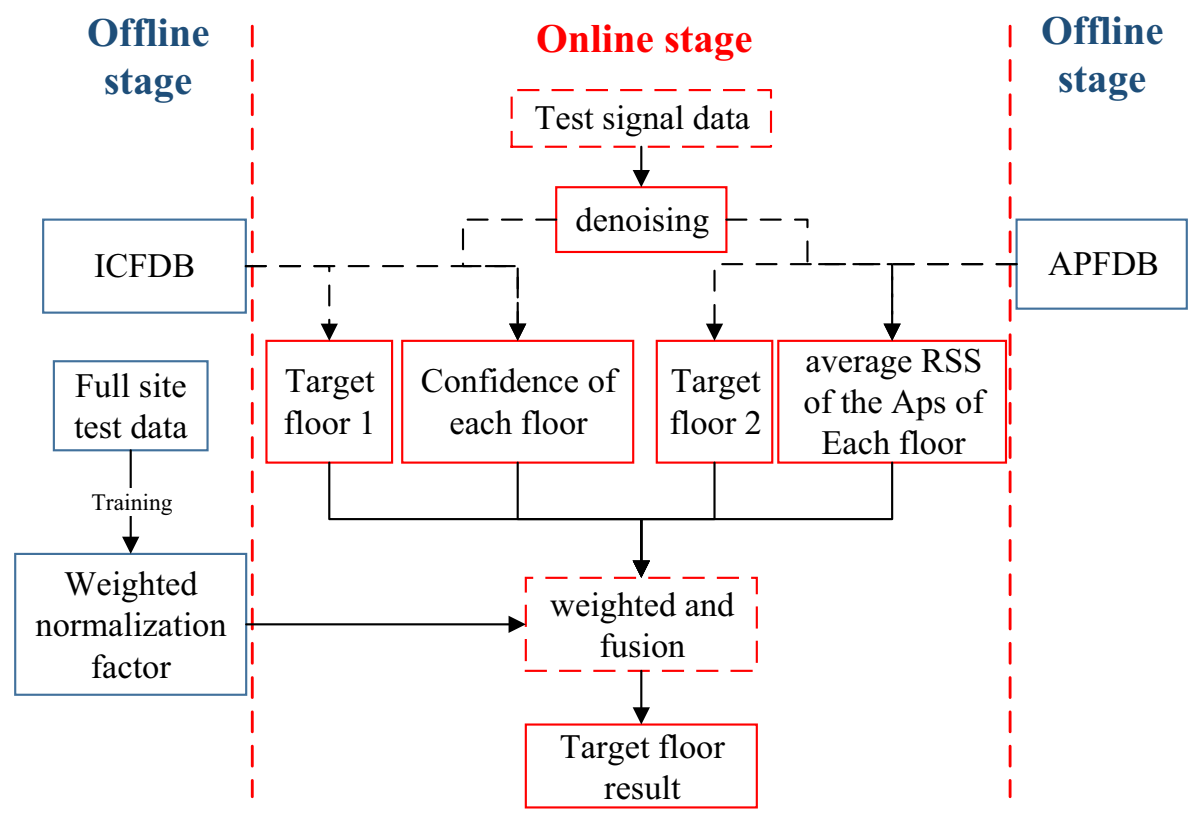

Fig. 10 Flowchart of the BLE floor positioning algorithm for multi-algorithm fusion

$$
\mathrm{TF}_{\text {test }}^{\prime}= \begin{cases}{\left[\mathrm{Mac}_{\mathrm{i}}, \mathrm{rssi}_{i}\right],} & \text { if } r s s i_{i} \geq-90 \mathrm{dBm} \\ {[\mathrm{null}],} & \text { if } r s s i_{i}<-90 \mathrm{dBm}\end{cases}
$$

According to statistics, the proportion of weak signal data that was eliminated was only about $3 \%$ of all the signals, and most of them were distributed in the farthest position from the AP. In practical applications, a variety of factors need to be considered in the signal rejection interval, including floor height, AP layout, laminate material and thickness, indoor area, and so on. For example, if the floor height is higher, the floor plank is thicker and the atrium space area is larger, then the attenuation of the wireless signal is more obvious. In this condition a high-precision floor positioning can also be achieved without removing weak signals.

\subsection{Floor positioning algorithm based on maximal average RSSI of the AP}

We improved the method algorithm from prior work (Maneerat et al. 2014a) by adopting BLE signals instead of Wi-Fi signals. Our method is affected by the number of APs allocated on each floor since floor positioning is conducted based on the average RSSI from each floor's APs. Assume that the number of BLE allocated on each floor is Mt, where $t=1,2, \ldots$, and $T$ denotes the floor. We used the notation $B F=\left[F_{t}, M a c_{t_{i}}\right]$, where $t_{-} i=1,2, \ldots$, and $\mathrm{Mac}_{t_{i} i}$ denotes the MAC address of the $i$-th BLE on the floor $t$ and the list of denoising fingerprints; and $\mathrm{TF}_{\text {test }}=\left[\mathrm{Mac}_{\mathrm{j}}, \mathrm{rssi}_{\mathrm{j}}\right]$, where $\mathrm{j}=1,2, \ldots, \mathrm{n}$, and $\mathrm{n}$ ' $<\mathrm{n}$ is the number of BLE after signal denoised. By matching TF' ${ }_{\text {test }}$ with the MAC addresses in BF and using the average RSSI of the AP on the same floor (named rssi_avg ${ }_{t}$ ), the target floor 
can be determined by finding the largest rssi_avg signal. The equation is as follows:

$$
\text { rssi_avg }{ }_{t}=\operatorname{average}\left(\operatorname{list}\left(\mathrm{rssi}_{j}, \quad \text { if } \operatorname{Mac}_{j} \in \mathrm{BF}\left(F_{t}, \operatorname{Mac}_{t_{-} i}\right)\right)\right.
$$

The target floor is $F_{\text {max_rss }}=\operatorname{argmax}\left(\mathrm{rssi}_{t}\right), \quad t=1,2, \ldots, T$

We summarized the target floor and the corresponding rssi_avg in the list $\mathrm{F} 1=\left[\mathrm{F}_{\text {max _rss }}\right.$, rssi_avg $]$, where $\mathrm{F}_{\text {max_rss }}$ denotes the first to the T-th floor in the test site, and rssi_avg $=\left[\right.$ rssi_avg $_{1}$, rssi_avg $_{2}, \ldots$, rssi_avg $\left._{\mathrm{T}}\right]$. The list was used as the evidence for assignments of weights and the indicator for integration of mixed floor positioning algorithms.

\subsection{Floor positioning algorithm based on signal confidence intervals}

The denoising test signals are used to continue the method. First, following the same protocol as prior work (Qi et al. 2019), we tested the fingerprint data and created a fingerprint database of the signal confidence intervals. We then used the denoised test fingerprint $\mathrm{TF}_{\text {test }}$ to conduct floor positioning.

Based on the original algorithm, we added a procedure of weight assignments and stored the confidence levels of different levels obtained in the last second step of the algorithm, which is denoted by $\mathrm{F} 2=\left[\mathrm{F}_{\text {interval }}\right.$, Interval $]$, where $\mathrm{F}_{\text {interval }}$ denotes from the first to the T-th floor; and Interval $=\left[\right.$ Interval $_{1}$, Interval $_{2}, \ldots$, Interval $\left._{\mathrm{T}}\right]$, which were used as the evidence for assignments of weights and the indicator for integration of algorithms to determine the target floor.

\subsection{Training and calculation of weight normalization factors}

We collected the data from the $\mathrm{K}$ test sites that were able to cover the entire test site environment. By applying the floor positioning algorithm from Chapters 3.2 and 3.3 of this paper, we obtained the results summarized in the two lists, $\mathrm{F}_{1}$ and $\mathrm{F}_{2}$. We then extracted the maximal and the second maximal values, denoted by $\max \left(\mathrm{rssi}_{-} \mathrm{avg}_{\mathrm{K}}\right), \max 1\left(\mathrm{rssi}_{\mathrm{avg}} \mathrm{K}_{\mathrm{K}}\right)$, $\max \left(\operatorname{Interval}_{\mathrm{K}}\right)$, and $\max 1\left(\operatorname{Interval}_{\mathrm{K}}\right)$ from rssi_avg and $_{\mathrm{K}}$ Interval ${ }_{\mathrm{k}}(\mathrm{k}=1,2, \ldots, \mathrm{K})$ at all the test sites according to the intermediate results rssi_avg $_{K}$ and Interval ${ }_{K}$ from the two lists. The initial weights of the two algorithms, $\mathrm{q}_{1 \mathrm{k}}$ and $\mathrm{q}_{2 \mathrm{k}}$, were calculated by Eq. (4) as follows:

$$
\left\{\begin{array}{l}
q_{1 k}=\left(\max \left(\operatorname{rssi}_{-} \operatorname{avg}_{k}\right)-\max 1\left(\text { rssi_avg }_{k}\right)\right) / \operatorname{abs}\left(\max \left(\operatorname{rssi}_{-} \operatorname{avg}_{k}\right)\right) \\
q_{2 k}=\left(\max \left(\operatorname{Interval}_{k}\right)-\max 1\left(\operatorname{Interval}_{k}\right)\right) / \max 1\left(\operatorname{Interval}_{k}\right)
\end{array}\right.
$$

After obtaining the initial weights of the two algorithms from Eq. (4), denoted by $\mathrm{q}_{1}=\left[\mathrm{q}_{11}, \mathrm{q}_{12}, \ldots, \mathrm{q}_{1 \mathrm{~K}}\right]$ and $\mathrm{q} 2=\left[\mathrm{q}_{21}, \mathrm{q}_{22}, \ldots, \mathrm{q}_{2 \mathrm{~K}}\right]$, we calculated the weight normalization factors $\mathrm{w}_{1}$ for $\mathrm{q}_{1}$ and $\mathrm{w}_{2}$ for $\mathrm{q}_{2}$ so that the two weights could have identical dimensionality. The two normalization factors satisfy the following relationship:

$$
\left\{\begin{array}{l}
w_{1}=1 \\
w_{2}=\operatorname{sum}\left(q_{1}\right) / \operatorname{sum}\left(q_{2}\right)
\end{array}\right.
$$

In the upcoming procedure of algorithm integration, the weights were to be normalized by the above factors, that is, multiplying the weights and the normalization factors, as shown in Eq. (6). 


$$
\left\{\begin{array}{l}
w_{1}^{\prime}=q_{1} * w_{1} \\
w_{2}^{\prime}=q_{2} * w_{2}
\end{array}\right.
$$

The use of the normalization factors $\mathrm{w}_{1}$ ' and $\mathrm{w}_{2}$ ' allowed the two methods to have identical dimensionality in the procedure of algorithm integration.

\subsection{Floor positioning by weighted integration}

Based on the floor results $\mathrm{F}_{\mathrm{t} 1}$ and $\mathrm{F}_{\mathrm{t} 2}$ obtained by the two floor positioning algorithms from Sects. 3.2 and 3.3, the intermediate values, rssi_avg ${ }_{\mathrm{t} 1}, \mathrm{rssi}_{\mathrm{i}} \mathrm{avg}_{\mathrm{t} 2}$, interval $\mathrm{t}_{\mathrm{t} 1}$ and interval $\mathrm{t}_{\mathrm{t} 2}$, corresponding to each algorithm are obtained. Formula (4) is used and the intermediate values are combined to generate the weights of the two results, and the calculation formula is as follows:

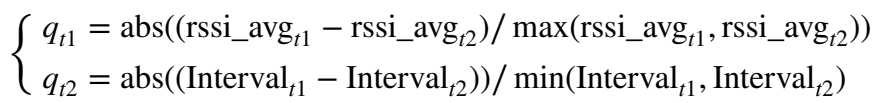

Then, the normalization factor trained in Sect. 3.4 adaptively normalizes the two weighted results as follows:

$$
\left\{\begin{array}{l}
w_{t 1}=q_{t 1} * w_{1}^{\prime} \\
w_{t 2}=q_{t 2} * w_{2}^{\prime}
\end{array}\right.
$$

Based on the two normalized new weights $\mathrm{w}_{\mathrm{t} 1}$ and $\mathrm{w}_{\mathrm{t} 2}$, the results of the two floors are merged and calculated and then rounded. Formula (9) gives the specific calculation process.

$$
F_{\text {result }}=\operatorname{round}\left(F_{t 1} * w_{t 1}+F_{t 2} * w_{t 2}\right)
$$

Finally, the rounded floor result is determined as the target floor, and the floor positioning algorithm is completed.

\section{Testing and analysis}

To facilitate the verification of the floor positioning performance of the methods, we used several methods that are more suitable for floor positioning at the test site. Besides, as each method requires, the corresponding fingerprint library was captured and created. At the same time, the same test data set was used as much as possible for assessment accuracy and performance analysis. The reference data used were as follows: Wi-Fi/BLE ICFDB, Wi-Fi/BLE APFDB, and Wi-Fi/BLE FFPDB. The test data was measured using Wi-Fi/ BLE FFPDB and FDTDB to compare the floor positioning accuracy between a 5-s static scenario and 1-s motion scenario. The 1-s motion data refers to the real-time test signal collected at $1 \mathrm{~Hz}$ by walking continuously for a period of time on each floor. The above data and a variety of algorithms were adopted to carry out floor positioning tests, and the accuracies of each method of floor positioning were compared. At the same time, a comparative analysis of each method's fingerprint database acquisition and maintenance workload as well as other indicators was conducted. Figure 3 shows that some of the strong 


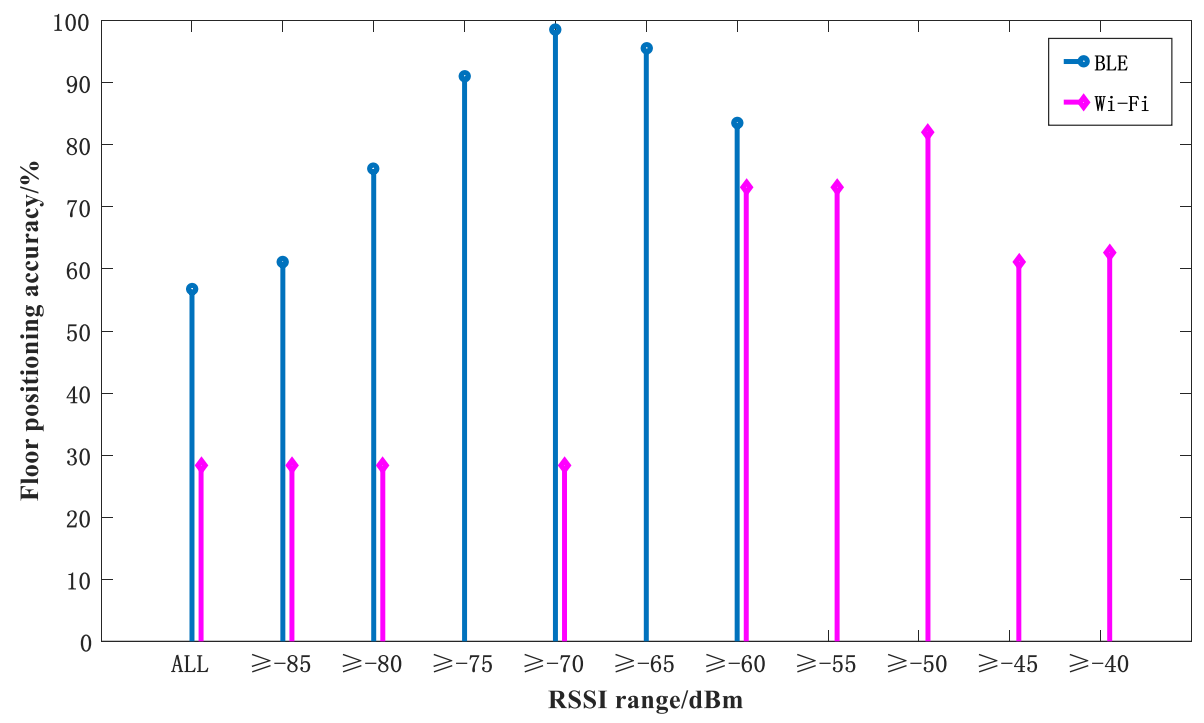

Fig. 11 Comparison of Wi-Fi/BLE floor positioning accuracy for different RSSI ranges in a multi-floor environment with an atrium space

signal data for the BLE signal reflected strong floor attributes, and most of the AP signals were distributed over the installation floor. A small percentage of the signal came from APs on adjacent floors, and a very small percentage of the signal came from APs two floors apart. The Wi-Fi signal had not only a strong RSSI but also large signal coverage throughout the three floors of the test site. As a result, Wi-Fi signals had weaker floor properties than BLE in the atrium space. Experiments were carried out based on the above wireless signal characteristics, and the results were compared and demonstrated.

\subsection{Comparison of Wi-Fi/BLE accuracy in determining floor positions based on the number of APs in different RSSI intervals}

Based primarily on the propagation characteristics of wireless signals that are located in a multi-floor environment with an atrium space, the number of APs on each floor based on different RSSI ranges can be counted to make decisions about the floor level. This method visually compares the floor positioning performance of Wi-Fi and BLE signals in such environments. The floor determination based on the number of APs can be described as follows: Using the 5-s FFPDB of Wi-Fi/BLE, the number of APs on each floor is associated with the Wi-Fi/BLE APFDB, and the floor with the largest number of APs is identified as the target floor. Three additional points are added here. The first one is that, if the number of APs deployed on each floor is different, then the ratio of the number of APs to the number of APs deployed on the current floor can be used as the eigenvalue for determination. Secondly, the fixed-point static signal is selected, and the 5-s RSSI mean value is taken, which can fully reflect the signal characteristics of the test location. Third, the use of different signal intervals for comparison can highlight the layered nature of $\mathrm{Wi}-\mathrm{Fi}$ and 
BLE signals. Different RSSI ranges correspond to different floor positioning accuracies, as shown in Fig. 11.

Figure 11 shows that the 5-s static BLE test signal had the highest floor location accuracy of $100 \%$ when the RSSI was greater or equal to $-70 \mathrm{dBm}$. The highest accuracy was only $82 \%$ when the Wi-Fi signal was at RSSI $\geq-50 \mathrm{dBm}$, and the Wi-Fi signal was generally less accurate than the BLE signal. It is also evident that the Wi-Fi signal was generally stronger than the BLE signal in terms of RSSI throughout the test site. Therefore, the Wi-Fi signals in the multi-floor environment of the atrium space structure could reach the floors at the more distant locations. The 5-s static test data was also not enough to improve the floor level determination accuracy of the Wi-Fi signal, which makes the use of BLE for floor positioning more ideal in this environment. Moreover, while the BLE signal in Fig. 11 reached $100 \%$ accuracy, this accuracy was achieved from test data that was collected in a 5-s static state. Figure 4 also shows that the BLE signal collected in real-time dynamics, that is, movement in $1 \mathrm{~s}$, was more volatile and had a smaller sample size. This does not guarantee that the BLE signal will always retain sufficient samples and achieve high accuracy when taking a value that is greater or equal to $-70 \mathrm{dBm}$. Therefore, if we want to obtain the highest floor positioning accuracy, the threshold range of the RSSI needs to be derived by a certain amount of professional training. Although this process is complicated, it is effective for most fingerprint-based methods, and thus we can consider whether to use it according to the actual situation. In this step, our method directly uses a robust method that removes a very small part (about 3\%) of the weakest signal.

\subsection{Performance analysis of multiple floor positioning methods}

To test the performance of the methods, we selected several floor positioning methods more suitable for comparison and analysis at the test site of this study. To ensure some degree of fairness, the algorithms were based on the same reference data and test data as much as possible. In terms of signals, there were two types of wireless signals, Wi-Fi and BLE. In terms of the collecting method, the test data was collected in two states: standing still for $5 \mathrm{~s}$ and moving in real-time for $1 \mathrm{~s}$. Several methods for locating the floors selected in this study are described below.

(1) K-nearest neighbor floor positioning algorithm. The steps of this algorithm were proposed by Alsehly et al. (2011). When the data from the fingerprint library of the 5-s fixed-point was tested, both Wi-Fi and BLE signals were selected with the scenario of $\mathrm{K}=1$ for comparison. Because the signal corresponding to the 5-s FFPDB can already represent the basic characteristics of the signal at the acquisition point, choosing reference points of the same density and using a longer period of time to acquire a reference fingerprint library will result in a significant difference in reference data from other methods. Moreover, this will result in an inability to reasonably compare floor positioning performance with other methods. In this case, we chose the source data of the slow-walking acquisition fingerprint database, known as the ICFDB, as the reference fingerprint.

(2) The strongest average RSSI floor positioning algorithm. This method is based on the ideas proposed by Bhargava et al. (2012), which "should select the relevant signal indicator attributes to determine the floor since the AP signal attenuates as it passes through the ceiling and floor" and "the average signal strength of APs from the same 
floor will be higher." The algorithm adopts the signal data collected at any TP, correlates the APFDB, counts the average signal value of APs on each floor, and eventually determines the floor where the AP group with the largest average signal value is located as the target floor.

(3) Signal interval confidence algorithm. This algorithm uses de-noised signals to carry out floor localization based on the method from Qi et al. (2019). The fingerprint data collected after a slow walk on each level was used to train the optimal signal intervals and finally aggregate to generate a Wi-Fi/BLE ICFDB, and we used FFPDB and FDTDB as the test data.

(4) Bayes algorithm. This method uses Bayes algorithm to carry out floor positioning. The method eliminates some of the weak signal APs from the TP data. It also chooses the APFDB as a reference. It selects the AP location as the eigenvalue instead of the RSSI. Furthermore, a Bayes algorithm calculates the probability that the test fingerprint belongs to each floor, and finally the location of the floor with the highest probability is determined as the target floor.

(5) The proposed method. The specific algorithmic process is explained in detail in Chapter 3 . In brief, it is a process of adding adaptive weighted integration to two methods to finalize the floor positioning results.

The same reference data was used here to generate a library of reference fingerprints in the format required for each method. Wherever possible, floor positioning was carried out using the same test data. The floor positioning performance of the five methods is shown in Table 5. As shown, the accuracy of the Wi-Fi signal floor positioning was lower than that of the BLE signal, except for the real-time dynamic test results of the K-nearest neighbor floor algorithm. However, among the five methods in a multi-floor environment, for the collection and maintenance, as well as the updating work of the fingerprint database on the floor nearest to $\mathrm{K}$, it was the most intensive one, and thus it is not suitable for general application in a large indoor multi-floor environment. Moreover, in the floor determination method using BLE signals, our method had the highest floor positioning accuracy in both state modes, that is, 5-s static and real-time dynamic. It also had a modest workload of fingerprint database collection, a small quantity of fingerprint library data collection, and a low complexity of the online phase algorithm. Furthermore, the general performance of the smartphone supports this as well. Besides, in the atrium multi-floor space, the floor recognition accuracy by the Bayes algorithm was no better than $15 \%$, which was described by Zhao et al. (2017). For these reasons, it is appropriate for our approach to be applied universally, especially in a multi-floor environment with an atrium space.

To more vividly show the floor positioning results of each algorithm in the atrium space environment, the floor positioning accuracy and the amount of fingerprint database data were taken as indicators and shown in a graphical display. In the test, the K-nearest neighbor and the proposed algorithm were used as examples to illustrate the fingerprint library acquisition workload. The K-nearest neighbor method requires the selection of multiple reference points, and reference points at the test site were established every $7-9 \mathrm{~m}$ on average. In that condition, we make the following assumptions: the entire floor could be laid out with an average of 12 reference points; for each reference point, only one direction was selected to collect the signal for $60 \mathrm{~s}$; there were 60 BLE APs in the whole test site; and the average signal coverage of each AP was about $70 \%$. As a result, the volume of the BLE signal fingerprint library data for the entire test site was 12 (reference points) * 3 (floors) $* 60$ (APs) $* 70 \%$ (AP coverage) $=1512$ pieces of data. On the other hand, the acquisition 


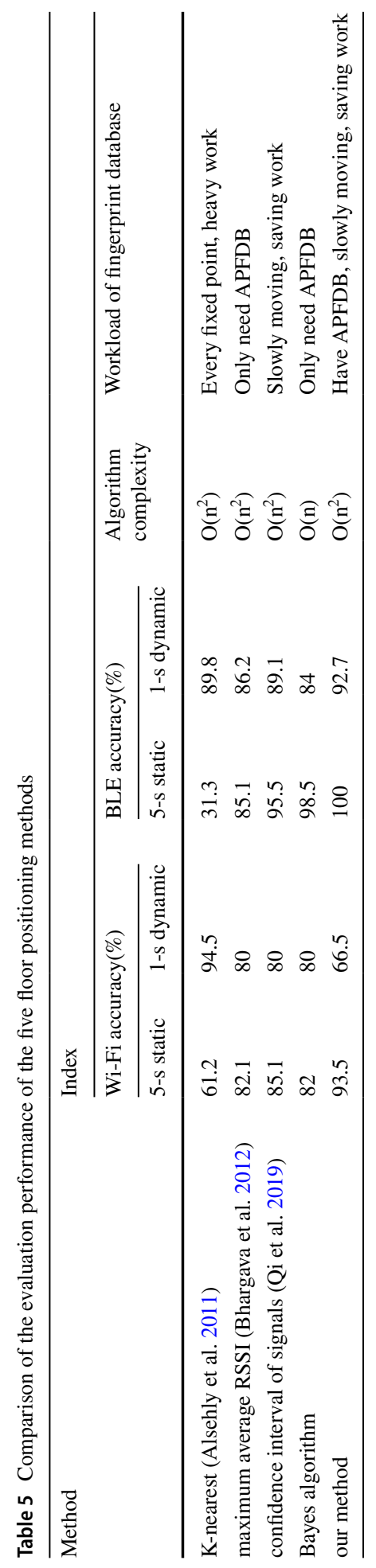




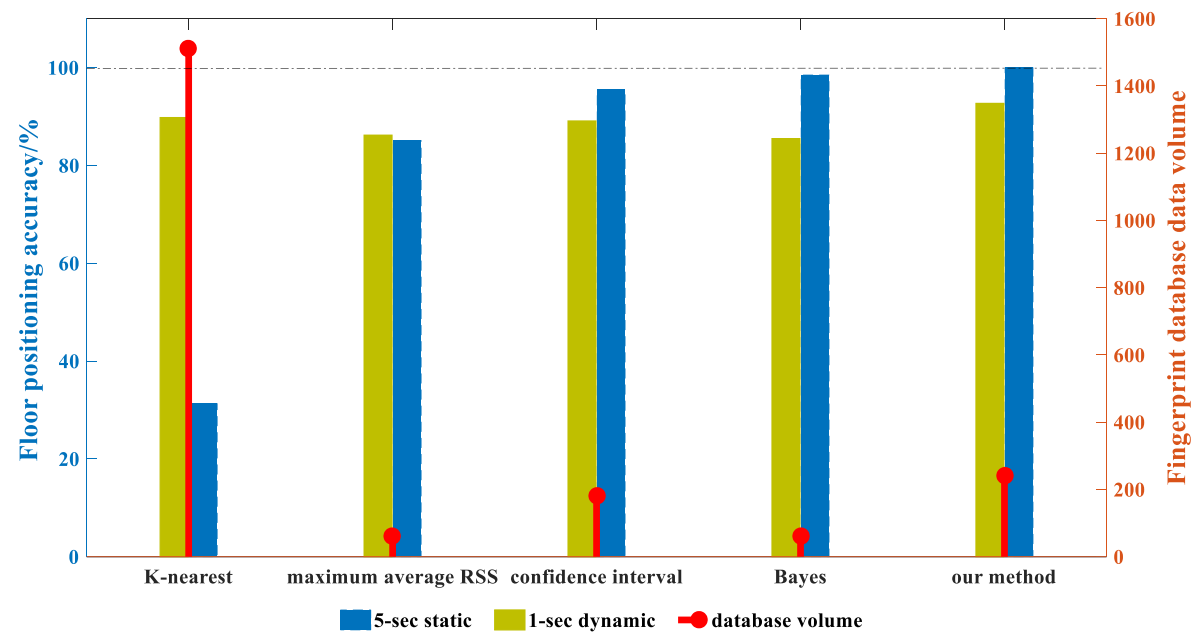

Fig. 12 Comparison of floor positioning accuracy and fingerprint database data volume by each method

time was 12 (reference points) $* 3$ (floors) $* 1$ (minute) +3 min of floor transition $=39$ min. In our algorithm, the amount of fingerprint library data collected by the signal ICFDB was about 3 (floors) $* 60$ (APs) $=180$ pieces of data plus 60 more AP layouts for a total of 240 pieces of data, and the fingerprint signal acquisition time for 3 times was 1.5 (minutes per floor) $* 3$ (floors) $* 3$ (times) $=13.5 \mathrm{~min}$. The most important difference between the two methods is that the amount of data in the fingerprint database based on the K-nearestneighbor floor positioning algorithm increases exponentially as the number of reference points and floor area increases, while the amount of data in the fingerprint database based on the method in this research is only a fraction of the increase. As the number of multifloor areas and floors increases, the difference in the amount of fingerprint library data between the two methods will become more pronounced. The performance of floor location based on the $\mathrm{K}$ nearest neighbor algorithm is restricted and is not applicable for a wide range of universal use.

The detailed data volume and positioning performance display is shown in Fig. 12. As shown, of the five BLE-based floor location algorithms, the proposed algorithm achieved the highest floor positioning accuracy in both 5-s static state and 1-s real-time dynamic motion. The amount of data in the fingerprint database was much smaller than that in an ordinary fingerprint database of pointing acquisition, and the overall positioning performance was optimal.

Moreover, during the 1-s real-time dynamic data test, the floor location accuracy of the method based on the confidence of the signal interval was $89.1 \%$ and the maximum mean signal floor AP was $86.2 \%$, which can be seen from Table 5. The result increased to $92.7 \%$ after adaptive weighted fusion of the two methods, which improved the overall floor positioning accuracy.

\subsection{Comparisons of floor positioning test results of multiple atrium space test sites}

To further test the performance of the method in this paper, we chose two other real multifloor experimental environments with atrium space, namely, an office building and the 


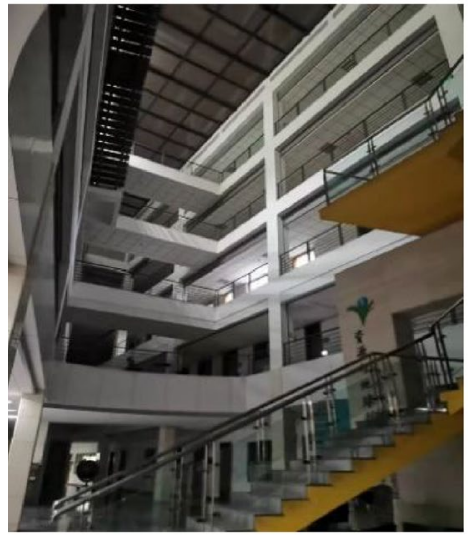

(a) office building F1

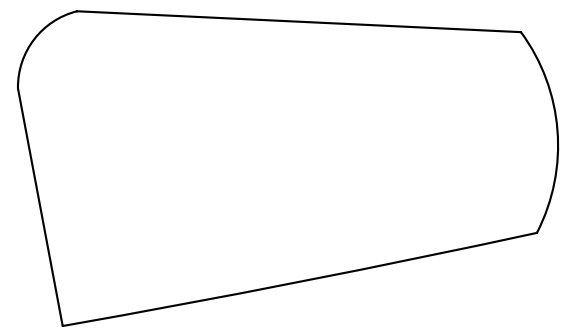

(c) schematic diagram F1

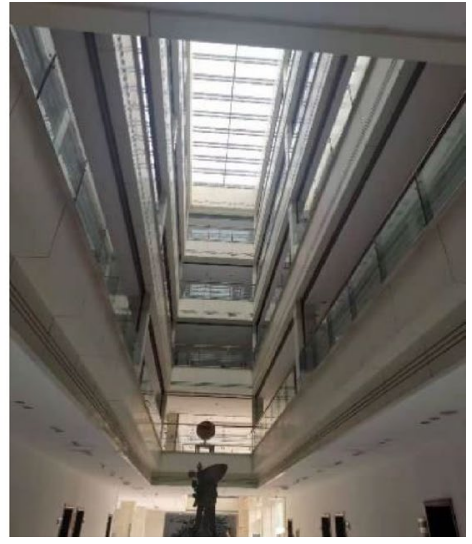

(b) administrative building F2

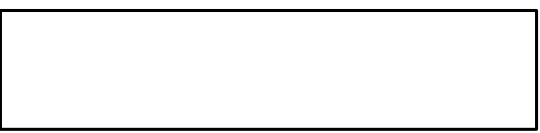

(d) schematic diagram F2

Fig. 13 Two additional real office building indoor environments and the corresponding schematic diagrams

administrative building of a university. Given the authenticity of the test site and site layout restrictions, we could not deploy additional Wi-Fi/BLE devices. The BLE beacon in the two test sites was so sparse that it could not support the floor positioning test. Meanwhile, the Wi-Fi signal density was higher than BLE, and it could basically meet our requirements; thus, we chose the Wi-Fi signal for the multi-method performance comparison. The specific indoor environment is shown in Fig. 13, where Fig. 13a, b represent the inner real scenes of a college building F1 and administrative building F2, respectively. Figure 13c, d are the schematic diagram of the atrium space area of Fig. 13a, b, respectively. Figure 13c, $\mathrm{d}$ show that the atrium space of the test site $\mathrm{F} 1$ is a polygonal irregular structure; the atrium space of the test site F2 is a slender rectangular structure. We used a variety of methods to compare floor positioning performance.

Given the actual test site layout conditions and the corresponding floor positioning method requirements, we employed several advanced machine learning classification methods to jointly carry out floor positioning tests at three test sites, C7, F1, and F2, and we compared the accuracy of the different methods. The methods included decision tree, SVM, KNN, and neural network. The specific floor positioning accuracy results are shown in Fig. 14. Each test site in the figure was tested for a 1-s motion state (1 s) and a 5-s static state ( $5 \mathrm{~s})$ test, and the FFPDB and FDTDB (1 s and $5 \mathrm{~s})$ used by each classification method were the same. As shown in the figure, except for the accuracy of the decision tree classification method in the 1-s real-time positioning test of the $\mathrm{C} 7$ test site that was 


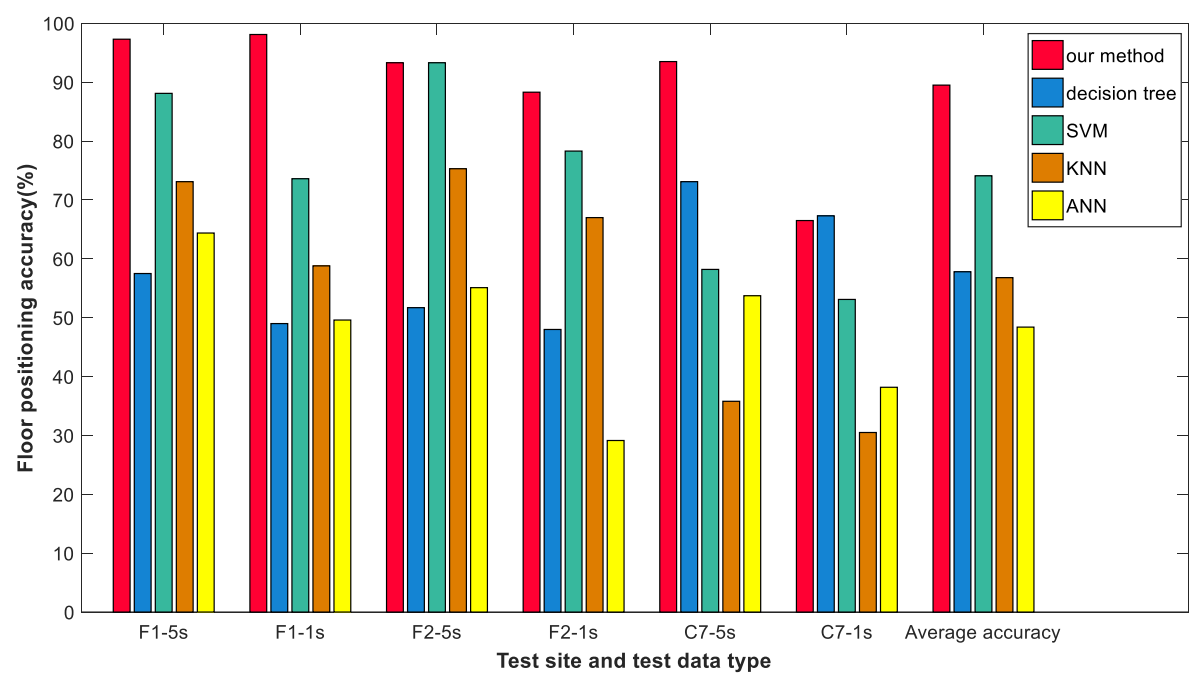

Fig.14 Comparison of the Wi-Fi floor positioning accuracy of multiple test sites and multiple methods

slightly higher than the method in this paper, the two methods with high accuracy of the test data in the other test sites were all the methods in our paper. The "average accuracy" in the figure represents the average value of the previous groups of histograms. Overall, the floor positioning accuracy of our method was better than the four other classification methods.

Moreover, among the above algorithms, the analyses can be summarized as follows: (1) The proposed algorithm requires a small amount of fingerprint data and location data to match and complete the floor positioning; meanwhile, without training the model, the algorithm efficiency is high, and the complexity is low. (2) The decision tree algorithm is more suitable for processing small sample size data, and classification training requires multiple scanning and sorting of the sample set, resulting in lower algorithm efficiency. (3) The SVM algorithm had better adaptability, higher resolution, and generalization performance, but it was sensitive to missing data, and the algorithm convergence time was not fast. (4) The KNN algorithm is simple and effective, and it relies on the limited nearby samples to complete classification. Meanwhile, the algorithm requires a large amount of calculation, pre-training, and determining the $\mathrm{K}$ value. Therefore, misjudging is easy in cases with a small sample size. (5) The ANN algorithm requires a large amount of calculation, the learning time is long, and the stability of the output results is low, which is not suitable for implementation in smartphones. In short, each algorithm has advantages and disadvantages. For the same training and test samples, the accuracy of various algorithms is shown in Fig. 14. In the training samples, 20-30 s of signal data were collected at each reference point, which was enough to reflect the signal characteristics at the collection location and ensure the performance of each classification algorithm. In general, the proposed algorithm performed well in the atrium spatial structure environment based on wireless signals in terms of computing performance and accuracy. 


\section{Conclusions}

Multi-floor environments in atrium spaces are common in the real-world context, but there are very few floor positioning methods specifically for this type of environment. In terms of the spatial propagation characteristics of the wireless signal, the biggest difference between this environment and an ordinary multi-floor setting is that the signal difference between floors is smaller. As a result, most floor positioning methods based on wireless signals do not work well in such environments. A BLE signal-based floor positioning algorithm is proposed for the first time specifically for multi-floor environments with an atrium space, and it was tested and verified. The proposed method not only solves floor location problems independently but also provides an initial floor reference for other relative floor positioning methods. This method can also be integrated with other methods of floor positioning or identifying floor changes, with a wide range of applications. It has a high accuracy of floor positioning and low cost as well as good universality.

This paper provides an in-depth analysis of the spatial propagation characteristics of $\mathrm{Wi}-\mathrm{Fi}$ and BLE signals in a multi-floor environment with an atrium space. It was found that the BLE signal performed significantly better than the Wi-Fi signal; therefore, the BLE signal was chosen for the floor positioning process in this environment. This research proposed a self-adaptation weighted integration of the BLE signal AP-based RSSI mean maximum floor and interval confidence floor determination method. Compared with other related methods, our method was found to have the highest floor positioning accuracy. The performance was tested in both of the two motion states. It was found that the floor positioning accuracy was up to $100 \%$ in the 5 -s static state; while the positioning accuracy was as high as $92.7 \%$ in the 1 -s real-time motion state, which is a significant improvement over the accuracy of the other four floor positioning methods. In terms of the workload of fingerprint database acquisition, the algorithm in this study saves both time and effort in acquiring the fingerprints. By walking slowly in the corridor area, the signal is captured with minimal volume of data, and the fingerprint database can be quickly captured and updated in case of any equipment changes.

There are some other things to consider. Regarding fingerprint library construction, the method in this paper was the same as the one based on wireless signal fingerprint positioning in most indoor positioning methods. They all involve the maintenance and updating of the fingerprint database. In practice, the timely updating of the fingerprint database needs to be considered. If the increase, removal, or displacement of positioning equipment is encountered, then a timely adjustment of the signal interval confidence fingerprint database to the AP floor positioning database is required. This work has been described in detail in related papers and will not be discussed here again. For the problem of heterogeneity of equipment, there is also special research detailing and solving this problem. The focus of this paper is on the methodical implementation of floor positioning. Moreover, to achieve both real-time effect as well as high accuracy, the rollback filtering of the real-time signal and the signal data of the first $\mathrm{n}$ seconds under the walking condition can be considered. After that, the floor determination is finally made by using the filtered signals. The accuracy can be further improved from the original 1-s real-time dynamics. The method in this study has been experimentally validated to achieve real-time floor positioning using $3 \mathrm{~s}$ of RSSI mean rollback data in motion, and its accuracy was as high as $98.2 \%$, which was improved by nearly $5 \%$ over the 1 -s data and was also in real-time.

Finally, it is difficult to directly obtain the location of the BLE beacon in reality, but there are many methods (Burgess et al. 2015; Gao et al. 2016; Abd Rahman et al. 2013) 
to be adopted mainly depending on the BLE signal characteristics to estimate the floor of the BLE, including the weighted centroid method (Lohan et al. 2017; Razavi et al. 2015). Even if there is no BLE deployment condition due to special circumstances, Wi-Fi signals can also be used instead, and, through the method steps analyzed in this paper, the floor positioning can be found in a multi-floor atrium space environment, and a satisfactory floor location estimate can be obtained.

Acknowledgements This work was supported by National Key Research and Development Program of China [grant numbers 2016YFB0502102, 2016YFB0502201]. This work was also supported by National Natural Science Foundation of China (No.42001397) and Introduction \& Training Program of Young Creative Talents of Shandong Province (0031802).

\section{References}

Abd Rahman MA, Dashti M, Zhang J (2013) Localization of unknown indoor wireless transmitter. In: International conference on localization and GNSS. IEEE, pp 1-6

Abd Rahman MA, Dashti M, Zhang J (2014) Floor determination for positioning in multi-story building. In: Wireless communications and networking conference. IEEE, pp 2540-2545

Alex V, Anthony LM, Jeffrey H, and Eyal de L (2007) The skyloc floor localization system. In: Proceedings of the fifth annual IEEE international conference on pervasive computing and communications. IEEE, pp 125-134

Alsehly F, Arslan T, Sevak Z (2011) Indoor positioning with floor determination in multi story buildings. In: International conference on indoor positioning and indoor navigation. IEEE, pp 1-7

Alshami IH, Ahmad NA, Sahibuddin S, Firdaus F (2017) Adaptive indoor positioning model based on WLAN-fingerprinting for dynamic and multi-floor environments. Sensors 17(8):1789

Ashraf I, Hur S, Shafiq M, Park Y (2019) Floor identification using magnetic field data with smartphone sensors. Sensors 19(11):2538

Bai Y, Jia W, Zhang H, Mao ZH, Sun M (2013) Helping the blind to find the floor of destination in multistory buildings using a barometer. In: Annual international conference of the IEEE engineering in medicine and biology society. IEEE, pp 4738-4741

Bhargava P, Krishnamoorthy S, Nakshathri AK, Mah M, Agrawala A (2012) Locus: an indoor localization, tracking and navigation system for multi-story buildings using heuristics derived from Wi-Fi signal strength. In: International conference on mobile and ubiquitous systems: computing, networking, and services. Springer, Berlin, Heidelberg. pp 212-223

Bi J, Wang Y, Li X et al (2018) A novel method of adaptive weighted K-nearest neighbor fingerprint indoor positioning considering user's orientation. Int J Distrib Sens Netw 14(6):1550147718785885

Burgess S, Åström K, Lindquist B, Ljungberg R, Sharma K (2015) Indoor localization using smartphones in multi floor environments without prior calibration or added infrastructure. In: International conference on indoor positioning and indoor navigation, pp 1-2

Campos RS, Lovisolo L, Campos MLRD (2014) Wi-Fi multi-floor indoor positioning considering architectural aspects and controlled computational complexity. Expert Syst Appl 41(14):6211-6223

Deng ZL, Wang WJ, Xu LM (2012) A K-Means based method to identify floor in WLAN indoor positioning system. Software 33(12):114-117

Elbakly R, Aly H, Youssef M (2018a) TrueStory: Accurate and robust RF-based floor estimation for challenging indoor environments. IEEE Sens J 18(24):10115-10124

Elbakly R, Elhamshary M, Youssef M (2018b) HyRise: A robust and ubiquitous multi-sensor fusion-based floor localization system. Proc ACM Interact, Mob, Wearable Ubiquitous Technol 2(3):1-23

Elbakly R, Youssef M (2020) The StoryTeller: scalable building-and AP-independent deep learning-based floor prediction. Proc ACM Interact, Mob, Wear Ubiquitous Technol 4(1):1-20

Fetzer T, Ebner F, Bullmann M, Deinzer F, Grzegorzek M (2018) Smartphone-based indoor localization within a 13th century historic building. Sensors 18(12):4095

Gansemer S, Hakobyan S, Püschel S et al (2009) 3D WLAN indoor positioning in multi-storey buildings. In: IEEE international workshop on intelligent data acquisition and advanced computing systems: technology and applications. IEEE, pp 669-672

Gao R, Zhao M, Ye T, Ye F et al (2016) Multi-story indoor floor plan reconstruction via mobile crowdsensing. IEEE Trans Mob Comput 15(6):1427-1442 
Gu F, Blankenbach J, Khoshelham K, Grottke J, Valaee S (2019) ZeeFi: Zero-effort floor identification with deep learning for indoor localization. In: Global communications conference. IEEE, pp 1-6

Gupta P, Bharadwaj S, Ramakrishnan S, Balakrishnan J (2014) Robust floor determination for indoor positioning. In: National conference on communications. IEEE, pp 1-6

Han L, Jiang L, Kong Q, Wang J, Zhang A, Song S (2019) Indoor localization within multi-story buildings using MAC and RSSI fingerprint vectors. Sensors 19(11):2433

Haque F, Dehghanian V, Fapojuwo AO, Nielsen J (2018) A sensor fusion-based framework for floor localization. IEEE Sens J 19(2):623-631

Honcharenko W, Bertoni HL, Dailing J (1993) Mechanisms governing propagation between different floors in buildings. IEEE Trans Antennas Propag 41(6):787-790

Huang J, Luo H, Shao W et al (2020) Accurate and robust floor positioning in complex indoor environments. Sensors 20(9):2698

Li B, Harvey B, Gallagher T (2014) Using barometers to determine the height for indoor positioning. In: International conference on indoor positioning and indoor navigation. IEEE, pp 1-7

Li Y, Gao Z, He Z, Zhang P, Chen R, El-Sheimy N (2018) Multi-sensor multi-floor 3D localization with robust floor detection. IEEE Access 6:76689-76699

Liu HH, Yang YN (2012) WiFi-based indoor positioning for multi-floor environment. In: TENCON 2011-2011 IEEE region 10 conference. IEEE, pp 597-601

Liu K, Motta G, Dong J (2017) Wi-Fi-aided magnetic field positioning with floor estimation in indoor multi-floor navigation services. In: International congress on internet of things. IEEE, pp 129-136

Lohan ES, Talvitie J, Silva PFE, Nurminen H et al (2015) Received signal strength models for WLAN and BLE-based indoor positioning in multi-floor buildings. In: International conference on location and GNSS. IEEE, pp 1-6

Lohan ES, Torres-Sospedra J, Leppäkoski H et al (2017) Wi-Fi crowdsourced fingerprinting dataset for indoor positioning. Data 2(4):32

Maneerat K, Prommak C, Kaemarungsi K (2014) Floor estimation algorithm for wireless indoor multistory positioning systems. In: International conference on electrical engineering/electronics, computer, telecommunications and information technology. IEEE, pp 1-5

Maneerat K, Prommak C (2014) An Enhanced floor estimation algorithm for indoor wireless localization systems using confidence interval approach. Int J Comput, Electr, Autom, Control Inf Eng 8(7):1182-1186

Maneerat K, Prommak C (2016) Floor determination algorithm with node failure consideration for indoor positioning systems. In: Proceedings of the 8th international conference on signal processing systems. pp 203-207

Peral-Rosado JAD, Bavaro M, López-Salcedo JA, Seco-Granados G, Chawdhry P, Fortuny-Guasch J et al (2015) Floor detection with indoor vertical positioning in LTE femtocell networks. In: Globecom workshops. IEEE, pp 1-6

Qi H, Wang Y, Bi J, Cao H, Si M (2019) Fast floor identification method based on confidence interval of Wi-Fi signals. Acta Geod Geophys 54(3):425-443

Ramana KV, Jianwei N, Aziz MAA, Umair MY (2016) A robust multi-cue blending-based approach for floor detection. In: International Bhurban conference on applied sciences and technology. IEEE, pp $647-653$

Razavi A, Valkama M, Lohan ES (2015) K-means fingerprint clustering for low-complexity floor estimation in indoor mobile localization. In: Globecom workshops. IEEE, pp 1-7

Razavi A, Valkama M, Lohan ES (2016) Robust statistical approaches for RSS-based floor detection in indoor localization. Sensors 16(6):793

Retscher G (2007) Augmentation of indoor positioning systems with a barometric pressure sensor for direct altitude determination in a multi-storey building. Cartogr Geogr Inf Sci 34(4):305-310

Shi J, Shin Y (2013) A low-complexity floor determination method based on WiFi for multi-floor buildings. In: Proceedings of the 9th advanced international conference on telecommunications, Rome, Italy. Vol: 2328

Sun L, Zheng Z, He T, Li F (2015) Multifloor Wi-Fi localization system with floor identification. Int J Distrib Sensor Netw 11(7):131523

Wang HY, Zheng VW, Zhao J, Yang Q (2010) Indoor localization in multi-floor environments with reduced effort. In: International conference on pervasive computing and communications. IEEE, pp 244-252

Xia H, Wang X, Qiao Y et al (2015) Using multiple barometers to detect the floor location of smart phones with built-in barometric sensors for indoor positioning. Sensors 15(4):7857-7877

Xu Z, Wei J, Zhu J, Yang W (2017) A robust floor localization method using inertial and barometer measurements. In: International conference on indoor positioning and indoor navigation. IEEE, pp 1-8 
Ye H, Gu T, Tao X, Lu J (2016) Scalable floor localization using barometer on smartphone. Wirel Commun Mob Comput 16(16):2557-2571

Ye H, Gu T, Tao X, Lu, J (2014) F-Loc: Floor localization via crowdsourcing. In: international conference on parallel and distributed systems. IEEE, pp 47-54

Ye H, Gu T, Zhu X et al (2012) FTrack: infrastructure-free floor localization via mobile phone sensing. In: International conference on pervasive computing and communications. IEEE, pp 2-10

Zhang S, Guo J, Wang W, Hu J (2018) Floor recognition based on SVM for WiFi indoor positioning. In: China satellite navigation conference. Springer, Singapore. pp 725-735

Zhao F, Luo H, Zhao X et al (2017) HYFI: hybrid floor identification based on wireless fingerprinting and barometric pressure. IEEE Trans Industr Inf 13(1):330-341

Zheng Z, Chen Y, Chen S, Sun L, Chen D (2016) Bigloc: a two-stage positioning method for large indoor space. Int J Distrib Sens Netw 12(6):1289013 